\title{
EXPERIMENTAL STUDIES OF PION-NUCLEUS INTERACTIONS AT INTERMEDIATE ENERGIES
}

\author{
Annual Progress Report \\ on DOE Grant No. DE-FG04-88ER40403
}

New Mexico State University

Las Cruces, NM 88003

December 31, 1992
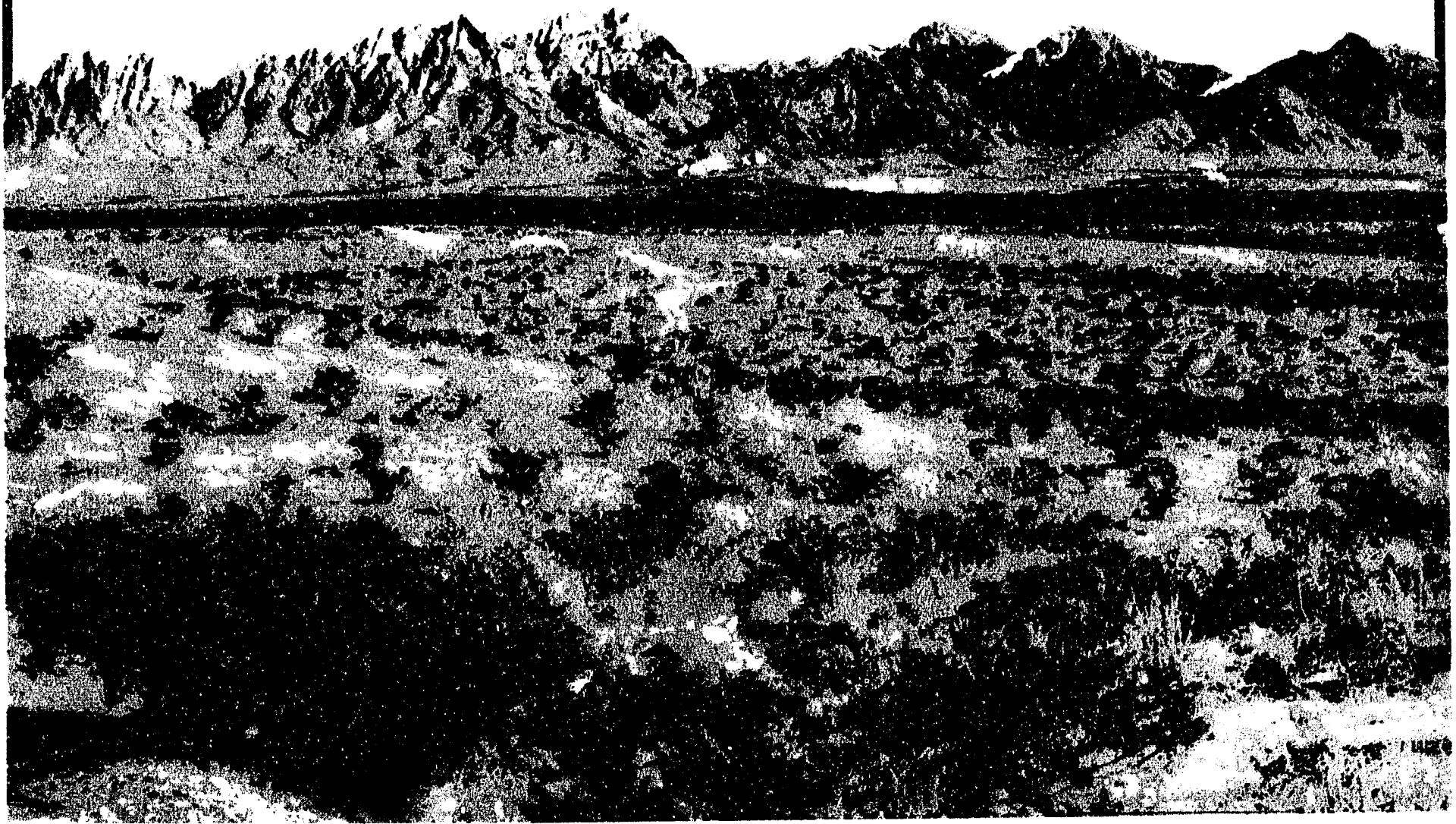

ILUISILUTION OF THIS DOCUMENT IS UPLLMITEO 


\title{
EXPERIMENTAL STUDIES OF PION-NUCLEUS INTERACTIONS AT INTERMEDIATE ENERGIES
}

\author{
Annual Progress Report \\ on DOE Grant No. DE-FG04-88ER40403
}

\author{
New Mexico State University \\ Las Cruces, NM 88003
}

December 31, 1992

\section{DISCLAIMER}

This report was prepared as an account of work sponsored by an agency of the United States Government. Neither the United States Government nor any agency thereof, nor any of their employees, makes any warranty, express or implied, or assumes any legal liability or responsibility for the accuracy, completeness, or usefulness of any information, apparatus, product, or process disclosed, or represents that its use would not infringe privately owned rights. Reference herein to any specific commercial product, process, or service by trade name, trademark, manufacturer, or otherwise does not necessarily constitute or imply its endorsement, recommendation, or favoring by the United States Government or any agency thereof. The views and opinions of authors expressed herein do not necessarily state or reflect those of the United States Government or any agency thereof.

$$
\text { Bing }
$$




\section{Contents}

1 Introduction 3

2 Experimental Research 4

2.1 Experiments Run . . . . . . . . . . . . . . . . . . 4

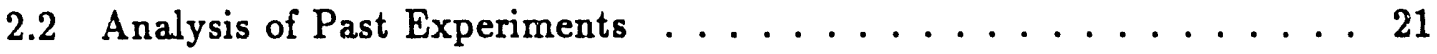

2.3 Preparation for Future Experiments . . . . . . . . . . . 25

3 Publications 36

3.1 Published Papers . . . . . . . . . . . . . . . . 36

3.2 Papers Submitted for Publication ................ 37

3.3 Papers Presented at Meetings and Conferences . . . . . . . . . . 37

4 Personnel $\quad 40$ 


\section{Introduction}

This report summarizes the work on experimental research in intermediate energy nuclear physics carried out at New Mexico State University in 1992 under a grant from the U. S. Department of Energy.

Most of these studies involved investigations of various pion-nucleus interactions and nucleon-nucleus charge-exchange reactions. The work was carried out with the LAMPF accelerator at the Los Alamos National Laboratory and the cyclotrons at the Paul Scherrer Institute (PSI) near Zurich, Switzerland, and at Indiana University (IUCF), as a a collaborative effort among several laboratories and universities. The NMSU personnel included two faculty members, George R. Burleson and Gary S. Kyle; two postdoctoral research associates, Maozhi Wang and Brent Park; and five graduate students, Zhinan Lin, Qihua Zhao, Brian Lail, Sean Blanchard, and Tinghua Chang.

The experimental activity at LAMPF involved measurements of new data on pion double-charge-exchange scattering, some initial wrok on a new Neutral Meson Spectrometer system, a search for deeply-bound pionlc atoms, measurements of elastic scattering, and studies of the $(n, p)$ reaction on various nuclei. At PSI we carried out measurements of pion quasielastic scattering, with detection of the recoil proton, on polarized ${ }^{7} \mathrm{Li}$. We also continued work on the analysis of data from a previous experiment at PSI on pion absorption in nuclei. This experiment involved using a detector system which we helped construct that covered nearly the full solid angle. In addition, we worked on plans and preparations for several experiments, including measurements of the asymmetry of low-energy pions scattered from polarized protons, measurements of single charge exchange scattering on ${ }^{13} \mathrm{C}$ and polarized ${ }^{3} \mathrm{He}$ with the new Neutral Meson Spectrometer system at LAMPF, measurements of pion elastic scattering from polarized ${ }^{3} \mathrm{He}$, and measurements of $(\mathrm{p}, \mathrm{n})$ reactions at IUCF and pion absorption studies at TRIUMF. These studies are aimed at improving our understanding of the pion-nucleus and nucleon-nucleus interaction mechanisms and of nuclear structure. 


\section{Experimental Research}

During this year we have been involved in data taking on several experiments in pion-nucleus and nucleon-nucleus interactions at LAMPF, PSI and IUCF, as well as in the analysis and interpretation of data from past experiments and the planning and preparation for future experiments. Our progress in this work is described below.

\subsection{Experiments Run}

In 1992 we were involved in data-taking runs on experiments at LAMPF on doublecharge-exchange and elastic scattering, a search for deeply-bound pionic atoms, some initial measurements of pion charge-exchange scattering with a new detector system, and measurements of nucleon-nucleus charge-exchange reactions; at PSI on measurements of the asymmetry of pion quasielastic scattering from polarized ${ }^{7} \mathrm{Li}$, and at IUFC on $(p, n)$ reactions. These include the following:

LAMPF Experiment No. 1107, Studies of Pion Double Charge Exchange Scattering at Energies above the $\Delta$ Resonance, University of Texas, University of Pennsylvania, Rutgers, University of York (UK), LANL, and NMSU (Park, Blanchard, Lail, and Burleson); G. Burleson, Spokesman.

This experiment, together with a companion experiment invol :ng measurements of elastic scattering, is a continuation of work begun by members of this collaboration which obtained the first extensive set of measurements of pion-nucleus interactions at energies immediately above the $\Delta(1232)$ resonance. The principal motivation for this is based on the observation that the pion-nucleon interaction is considerably weaker at these energies than near the peak of the $\Delta$ resonance. This means that the resulting longer mean free paths for pions in nuclei and the weaker optical potentials should lead to more reliable theoretical calculations. These energies also have advantages over those below the resonance, where the interaction is also weaker, but where second-order effects are larger.

Double-charge-exchange (DCX) scattering is of particular interest because it must take place on at least two nucleons and therefore should be able to give new insight into nucleon-nucleon correlations. In order to carry out detailed calculations of DCX at these energies, however, it is necessary to know how well models of the pion-nucleus interaction can reproduce simpler interactions, such as elastic scattering. Partly for this reason, measurements were carried out on both of these processes.

Results for transitions to double isobaric analog states (DIAS) have been published. $[1,2,3]$ They have generally disagreed with most previous expectations. The 
agreement with new calculations of Oset and Strottman, [4] which include strong isovector medium polarization effects, appear to be very good, however.

New results on the nonanalog transition in ${ }^{16} \mathrm{O}$ to the ground state of ${ }^{16} \mathrm{Ne}$ are shown in. Fig. 1. The interest in nonanalog transitions at these energies lies in the observation that at resonance energies they are very well described by the Delta interaction (DINT) model, [6] while analog transitions seen to be consistent with a sequential mechanism. An attempt to describe DCX scattering with a combination of these two mechanisms has been unsuccessful, however. $[7,8]$ At energies above 300 $\mathrm{MeV}$, the DINT mechanism predicts a very small cross section, while preliminary indications suggest a larger one. The results shown in Fig. 1 indicate that the cross sections at these energies are larger than those between 200 and $300 \mathrm{MeV}$, suggesting that another mechanism besides DINT is present at these higher energies. These data will be included in a Ph.D. thesis of David Beatty, of Rutgers University.

In 1992 we measured angular distributions for $\mathrm{DCX}$ on ${ }^{44,48} \mathrm{Ca}$ at $400 \mathrm{MeV}$. Previous data on these nuclei, along with other nuclei in the $f_{7 / 2}$ shell, have been interpreted in terms of a two-amplitude model involving transitions through analog and nonanalog states.[9] This model has been very successful at low energies,[10] but it was found to be less successful at these higher energies.[2, 3] At low energies, the angualr distributions of these nuclei were found to be very different,[10] with that for ${ }^{48} \mathrm{Ca}$ being flat and ${ }^{44} \mathrm{Ca}$ forward peaked. Preliminary results indicate that the two have similar shapes at these higher energies and resemble very much predictions for the ${ }^{42} \mathrm{Ca}$ angular distribution of Oset and Strottman.[11]

LAMPF Experiment No. 1140, Search for $\eta$ Component in Pion DCX Scattering, University of Texas, University of Minnesota, University of Pennsylvania, University of York (UK), King Fahd University (Saudi Arabia), LANL, and NMSU (Rawool-Sullivan, El-Ghossain, and Burleson); C. L. Morris, LANL, and C. F. Moore, Texas Spokesmen.

Calculations by Haider and Liu[12] have shown that a bound state of an $\eta$ in a nucleus may occur as an intermediate state in pion DCX. Such an effect would compete with $\pi^{0}$ and $\eta$ channels in the continuum and would lead to a resonance-like structure in the DCX excitation function at fixed momentum transfer, due to interference between these channels. Calculations for the reaction ${ }^{14} \mathrm{C}\left(\pi^{+}, \pi^{-}\right){ }^{14} \mathrm{O}$ (DIAS) predict that this structure should occur with a fluctuation of $\sim 80 \%$ at a momentum transfer of $210 \mathrm{MeV} / \mathrm{c}$. For the ${ }^{18} \mathrm{O}\left(\pi^{+}, \pi^{-}\right)^{18} \mathrm{Ne}$ reaction, the resonance effect should be seen at about $420 \mathrm{MeV}$.

Data were taken on the ${ }^{18} \mathrm{O}$ reaction at fixed momentum transfers of $\sim 0,105$, and $210 \mathrm{MeV} / \mathrm{c}$, for pion energies between 350 and $440 \mathrm{MeV}$. Most of the work was done in 1991, but some additional measurements were made in 1992 . A plot 


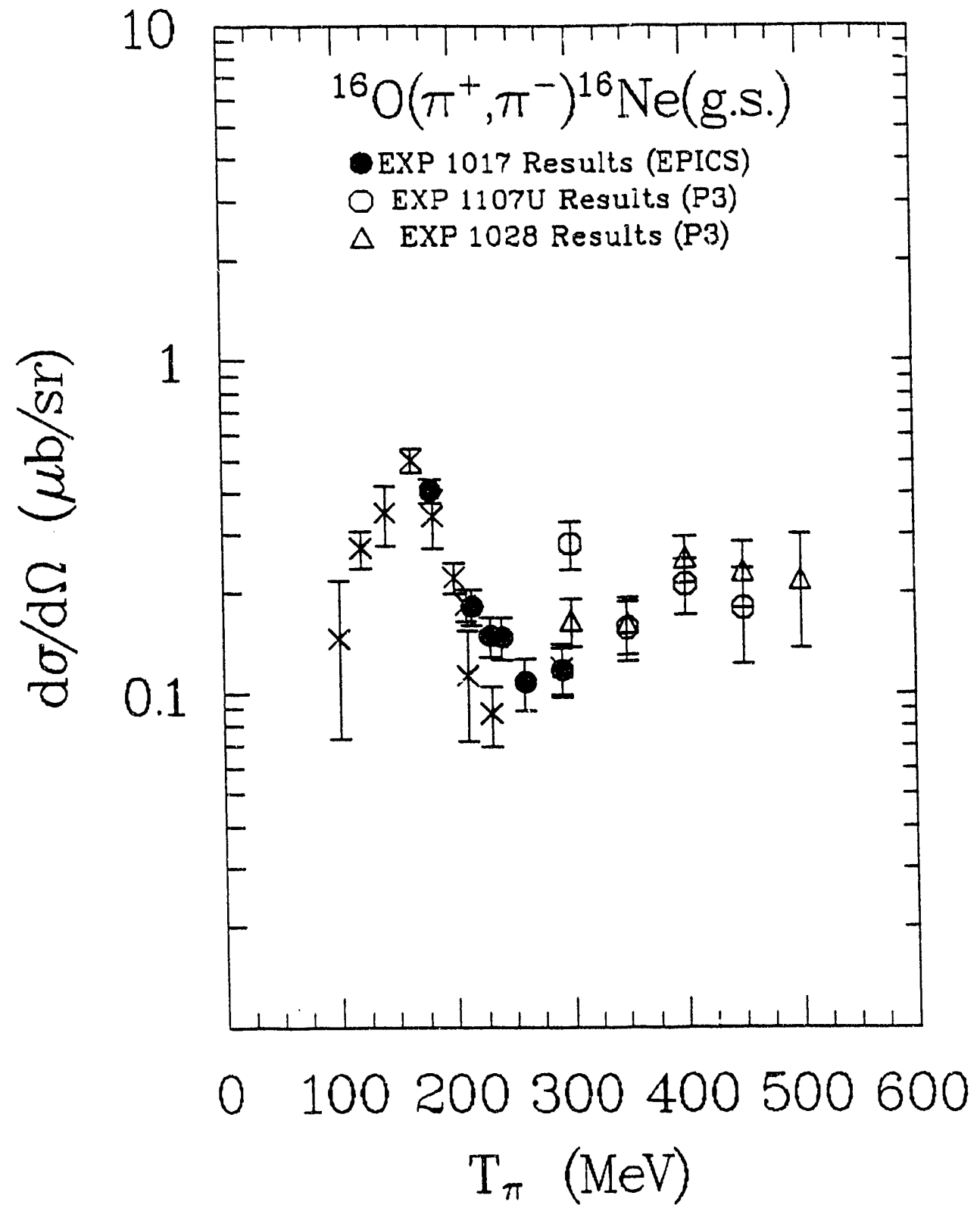

FIG. 1. Forward-angle differential cross sections from LAMPF Exp. 1107 for the pion double charge reaction on ${ }^{16} \mathrm{O}$ leading to the ground state of ${ }^{16} \mathrm{Ne}$, a nonanalog transition, compared with previous results. The data marked with crosses are from ref.[5], and the remainder are unpublished. 
of some of the results is shown in Fig. 2, in the form of a ratio of the $q=210$ and $\mathrm{q} \sim 0$ cross sections for the analog state transition as well as for the continuum region. The lines shows represent straight-line fits to the data. While the continuum region shows no evidence of structure, the DIAS transition suggests the presence of structure at the expected energy, though the statistical precision of the data is not sufficient to allow more than a qualitative characterization of this effect. These results have been submitted for publication and should form the basis of a Ph.D. thesis for John Johnson, of the University of Texas.

LAMPF Experiment No. 1216, The ${ }^{2} \mathrm{H},{ }^{3} \mathrm{He},{ }^{4} \mathrm{He}\left(\pi, \pi^{\prime} x\right)$ Reaction, University of Minnesota, Rutgers, LANL, University of Texas, University of Colorado, Tohuko University, and NMSU (Park); J. Langenbrunner, and D. Dehnhard, Minnesota, and M. K. Jones, Rutgers, Spokesmen.

Exclusive experiments on few-body nuclei ${ }^{2} \mathrm{H},{ }^{3} \mathrm{He}$, and ${ }^{4} \mathrm{He}$ are motivated in part by the surprisingly large ratio of the cross sections $R=\frac{\sigma\left(\pi^{+}, \pi^{+} p\right)}{\sigma\left(\pi^{-}, \pi^{-1} p\right)}$. Jones $e^{t}$ al. [13] have found $R \geq 25$ in the region of nuclear excitation energy near $30 \mathrm{MeV}$. In sharp contrast, in the same region of nuclear excitation, the ratio of inelastic cross sections give the expected response $\frac{\sigma\left(\pi^{+}, \pi^{+1}\right)}{\sigma\left(\pi^{-}, \pi^{-1}\right)} \cong 1$.

If the ${ }^{A} \mathrm{He}\left(\pi, \pi^{\prime} p\right)$ reaction were to proceed exclusively by quasi-elastic $\pi$-proton scattering, we would expect $R=9$ because the ratio of $\pi^{+}$-proton to $\pi^{-}$-proton elastic scattering amplitudes is 3 for the $P_{33}$ resonance. However, for the decay of a state of good isospin, this ratio should be near unity.

The ${ }^{16} \mathrm{O}\left(\pi^{ \pm}, \pi^{ \pm \prime} p\right)$ reaction showed a similar phenomenon in terms of this ratio.[14] Takaki and Thies et al [15] have modeled the ${ }^{16} \mathrm{O}\left(\pi^{ \pm}, \pi^{ \pm \prime} p\right)$ reaction by including a $\Delta-N$ interaction. In that model, a $\Delta$-induced proton knockout amplitude interferes with the quasi-free proton knockout process ("medium effects"). Understanding such a phenomenon necessarily involves understanding the role of the nuclear medium, and perhaps the role of nuclear excitations. Therefore we undertook to measure these ratios for the simplest few-body nuclei, where much progress has been made in the quantitative understanding of the nuclear wavefunctions.[16]

The data analysis is in progress and will be used as a part of Ph.D. requirement for C. Whitley of the University of Texas.

LAMPF Experiment No. 1220, Measurement of Cross-Section Angular Distribution for the ${ }^{6} \mathrm{Li}\left(\pi^{+}, \pi^{+}\right)$at $T_{\pi}=100,134,164,190$ and $219 \mathrm{MeV}$, University of Karlsruhe, University of Minnesota, Rutgers, LANL, and NMSU (Park); E. Boschitz aud B. Brinkmöller, Karlsruhe, Spokesmen.

The ${ }^{6} \mathrm{Li}$ nucleus shows typical properties of heavier nuclei but is light enough to allow the calculation of nuclear processes with realistic interaction mechamisms. 


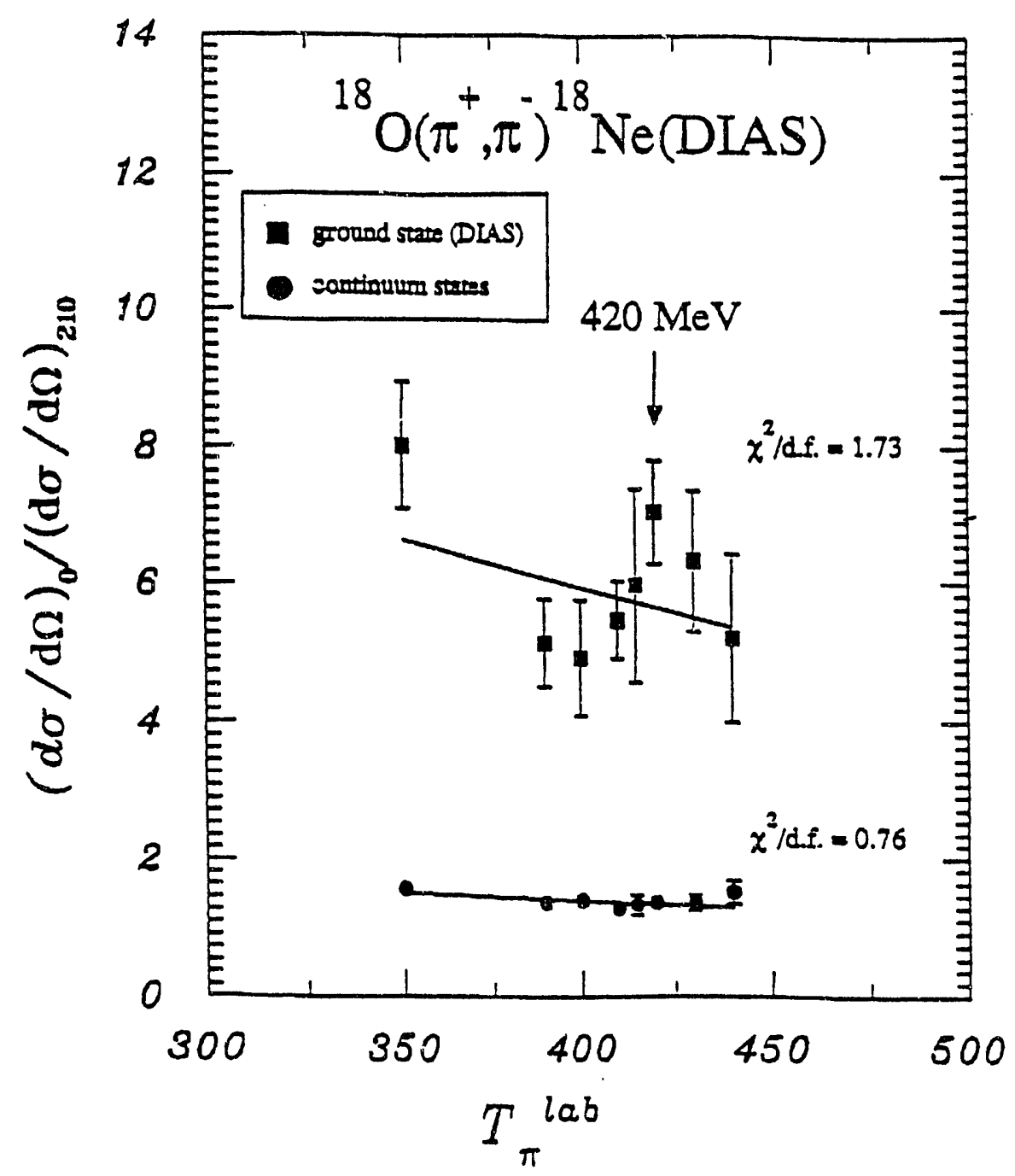

FrG. 2. Ratios of the $q=210$ and $q \sim 0$ cross sections for the ${ }^{18} \mathrm{O}\left(\pi^{+}, \pi^{-}\right)^{18} \mathrm{Ne}$ (DIAS) reaction as a furction of pion energy, from LAMPF Exp. 1140. The lines represent the best fits of straight lines to the data, with values of $\chi^{2}$ per degree of freedom indicated. The fluctuation at $420 \mathrm{MeV}$ suggests the structure expected as a signature of a bound $\eta$ in a nucleus. 
Polarized data at the proposed energies (with an exception of $190 \mathrm{MeV}$ ) have been previously taken at PSI;[17] however, very good knowledge of differential cross section is needed to put tighter constraints on the models that are used to describe the results. Therefore we have made precise cross-section measurement over an angular range between $30^{\circ}$ and $120^{\circ}$ in $1^{\circ}$ bins, which allowed us to measured the depth and shape of the first minimum. The results are currently being analyzed and will allow a more rigorous test of the theoretical models.

LAMPF Proposal No. 1239, Feasibility of the Direct Production of Pionic Atoms, California Polytechnic Institute, Rutgers, Arizona State University, Institute of Nuclear Research (Moscow), Academy of Sciences (Russia), LANL, and NMSU (Park); M. Rawool-Sullivan and C. L. Morris, LANL, and P. Siegel, California Polytechnic Institute, Spokesmen.

One quantity of particular interest in the pion-nucleus interaction is the threshold pion-nucleus amplitude, which has a bearing on chiral-symmetry breaking and on the anomalous behavior or the level shifts and widths of the energy levels of some pi-mesonic atoms. When a negatively-charged pion is captured by an atom, it normally is initially found in a highly-excited orbit and then de-excites through electromagnetic transitions. For light nuclei, it generally reaches the $1 \mathrm{~s}$ level, from which it annihilates on the nucleus. The measured $x$-ray spectrum gives information on the atomic energy levels, which in general are shifted from the pure Coulomb values by the strong interaction. In the lower angular-momentum states, these shifts are crucial in chararterizing the threshold pion-nucleus optical potential.

For larger nuclei, the lower-level pionic wave functions overlap the nucleus more completely, so that their sensitivity to the pion-nucleus interaction is greater. Experimental observations of the energies and widths of these states in these nuclei would provide important constraints on theoretical models. For these nuclei, however, these low leveis are seldom populated, since the pion is generally absorbed before it reaches them in a cascade process.

Several methods have been proposed for populating these states and determining their modification due to the strong interaction. One possibility is the $\left(\pi^{-}, p\right)$ channel, corresponsing to the incident pion being captured directly into a $1 s$ or $2 p$ atomic orbital after striking a proton and knocking it out of the nucleus. Calculations of cross sections for this process process, using the distorted-wave inpulse approximation, have been carried out by Kaufmann, et al,[18] which indicate that these cross sections are largest at small energies.

This year we attempted to look for a signal of the formation of this system by using this reaction with a ${ }^{58} \mathrm{Ni}$ target with $30-\mathrm{MeV}$ incident pions. Reaction products were detected with a set of 30 plastic-BGO phoswich detectors which 
covered nearly the full solid angle. The trigger for the experiment required the pion to disappear from the beam and two hits to appear in the detector system. The data are currently being analyzed.

LAMPF Experiment No. 1263, Analog Contribution to Nonanalog Double Charge Exchange, University of Pennsylvania, LANL, and NMSU (Park, Blanchard, and Lail); A. L. Williams and H. T. Fortune, University of Pennsylvania, Spokesmen.

In lowest order, DCX reactions are described in terms of two sequential single charge exchange (SCX) scatterings on two nucleons. For $\mathrm{T}=1 / 2$ nuclei, the $\left(\pi^{+}, \pi^{-}\right)$ reaction involves one scattering to analog states followed by a scattering to nonanalog states, or vice versa, since there is no transition route involving two sequential analog transitions. SCX data, which show strong peaks for transitions to the isobaric analog state (IAS), suggest that the sequence of an initial analog transition followed by a non-analog transition is the stronger. Measurements of DCX on a $T=1 / 2$ nucleus, when considered along with the single charge exchange to the IAS, should provide constraints on the nature of the non-analog charge exchange mechanism.

In this experiment, measurements of $\left(\pi^{+}, \pi^{-}\right)$on ${ }^{27} \mathrm{Al}$ were carried out at the EPICS channel. This target was chosen because of the existence of DCX data on the neighboring nuclei ${ }^{26} \mathrm{Mg}$ and ${ }^{28} \mathrm{Si}$, both of which exhibit behavior in the DCX reaction that is typical of other similar nuclei. It is expected that an analysis of cross sections of DCX on these nuclei will give more insight into the two-step DCX process.

LAMPF Experiment No. 1268, $\pi^{-} p \rightarrow \pi^{0} n$ Cross Sections in the Region of the $\Delta$ Resonance, NMS Collaboration, including NMSU (Park, Zhao); M. E. Sadler, Abilene Christian University, Spokesman.

This is one of two experiments run in summer, 1992, as part of the tuneup of the new Neutral Meson Spectrometer (NMS). This device is a new high-resolution, large-acceptance spectrometer for detecting neutral mesons which was built at at LAMPF, with contributions from several university groups. It is designed to detect the two-gamma decay of $\pi^{0}$ and $\eta$ mesons and should be able to have an energy resolution for the meson of better than $300 \mathrm{keV}$. It consists of two rectangular arrays of (pure) CsI scintillation counters for gamma-ray shower detection, preceeded by BGO converters with multiwire tracking chambers to determine the positions of the gamma rays. NMSU worked on tests of prototype photomultiplier tubes for the CsI counters, construction and tests of the multiwire chambers, and beam tests of parts of the system.

The purpose of this experiment is to measure $\pi^{-} p \rightarrow \pi^{0} n$ differential cross section in the region of the $\Delta(1232)$ resonance. The point of this is to check previous data, which do not agree well with partial wave analyses at back angles. The existing 
data have quoted errors of $6-10 \%$ at these angles, and all involved the use of neutron counters, which are sometimes prone to efficiency variations. A systematic measurements by one group of a complete angular distribution does not exist at any energy. With the NMS to detect $\pi^{0}$ mesons, a systematic set of high-quality data should be possible for the first time. This should provide input for charge-independent partialwave analyses which should be able to investigate sharge-symmetry breaking, the charge splitting of the $\Delta$, and to help clarify discrepancies at lower energies through analyticity constraints on the partial-wave analyses.

Some data was taken for this experiment in fall, 1992, as part of the NMS tuneup. Measurements were made of forward-angle cross sections at six energies between 142 and $266 \mathrm{MeV}$. Additional running is expected in 1993.

LAMPF Experiment No. 1288, Measurements of Differential Cross Sections for $\pi^{+}$Scattering from ${ }^{13} \mathrm{C}$ at $T_{\pi}=190$ and $229 \mathrm{MeV}$, University of Minnesota, Rutgers, LANL, and NMSU (Park); D. Dehnhard, Minnesota, Spokesman.

The first measurement of asymmetries of $\pi^{ \pm}$elastic scattering from polarized ${ }^{13} \mathrm{C}$ was done in 1989 with the Large Acceptance Spectrometer (LAS) at LAMPF.[19] In analyzing the data it became apparent that the acceptance of the LAS at large scattering angles was not sufficiently well known to extract reliable differential cross sections. However, for a thorough theoretical analysis of the asymmetry data it is important to have accurate differential ross section data as well. We therefore proposed to measure the differential cross secric is with the EPICS system.

The acceptance of EPICS is quite well known from many experiments and its better energy resolution permitted higher-quality data to be taken. The measurements were carried out during the last run cycle of 1992 at $\mathrm{T}_{\pi}=130$ and $223 \mathrm{MeV}$, the prviously-measured energies, but the allotted beam time was sufficient to take data only with $\pi^{+}$. Spectra were taken in $5^{\circ}$ steps from $25^{\circ}$ to $120^{\circ}$. The statistics accumulated were sufficient to use $1^{\circ}$-binning in replay.

Preliminary cross sections display well-defined first and second minima in the detailed angular distributions (see Fig. 3). There appears to be good agreement with the previous data at the forward angles. However, in the region of the second minimum the new data show systematically smaller cross sections at both energies. Since the cross-section measurements were performed with a slab target, where the resolution allowed a good separation between the ground state and first excited state, there appears to be a problem with the acceptance correction of the LAS at the large angles. The new data from EPICS should now allow a better analysis of the asymmetry data in order to test a variety of pion-nucleus reaction models.

LAMPF Experiment No. 823U, The $(n, p)$ Reaction at $800 \mathrm{MeV}$ as a Probe of Spin-Flip and Non-Spin-Flip Giant Resonances and the Quasi-Free Continuum, 


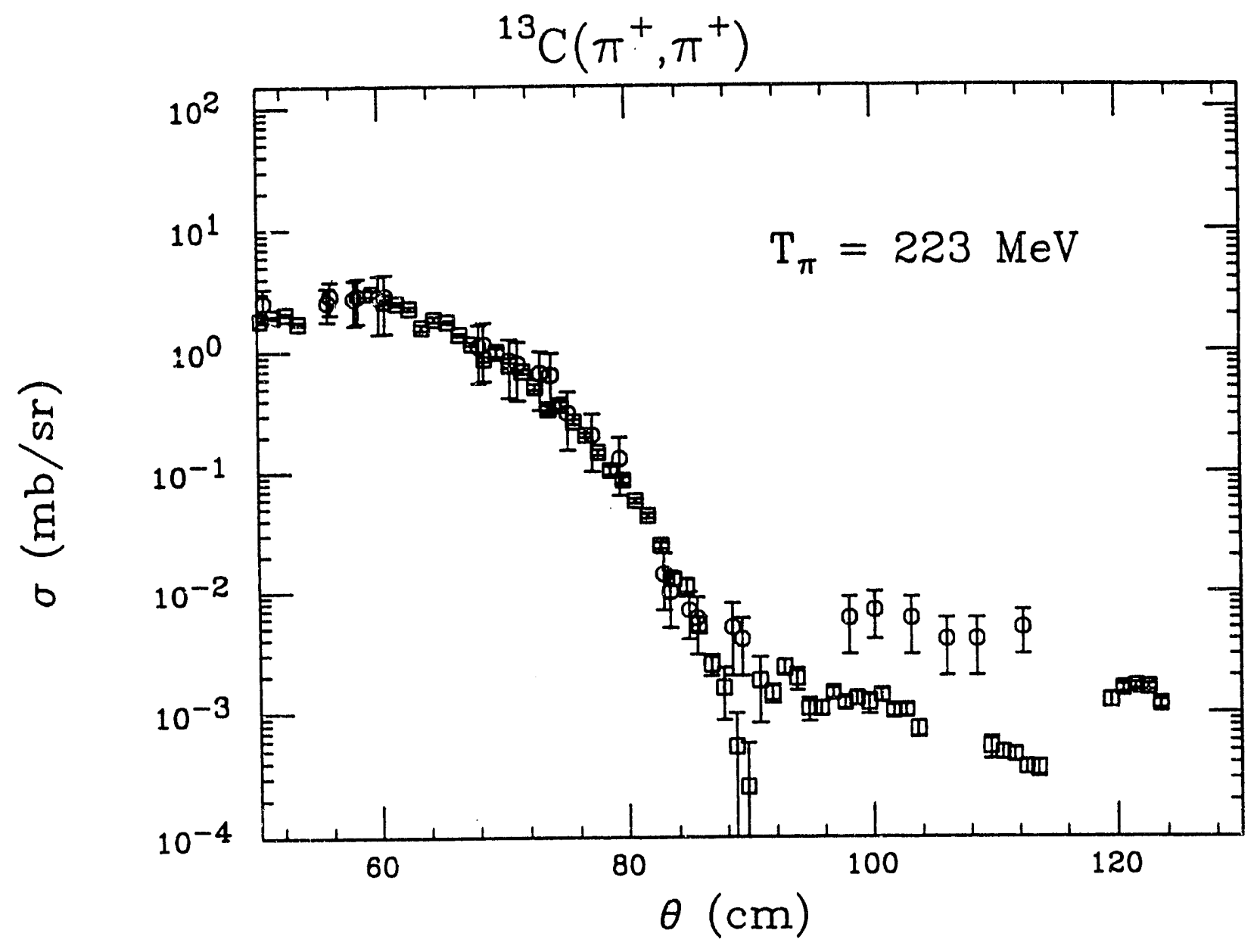

FIG. 3. Cross section angular distributions for the ${ }^{13} \mathrm{C}\left(\pi^{+}, \pi^{+}\right)$reaction from previous work and from LAMPF Exp. 1288 are shown with circles and squares, respectively. The new data show two very well-defined minima near $90^{\circ}$ and $116^{\circ}$ that are not seen in the previous data. 
Ohio University, Ohio State University, LANL, and NMSU (Park); J. L. Ullmann. N. S. P. King, and R. C. Haight, LANL, and J. Rapaport, Ohio University, Spokesmen.

This experiment was proposed several years ago and provided part of the impetus for building the Medium Resolution Spectrometer (MRS). The original proposal suggested a wide range of physics problems to be studied. One of the most interesting questions (Gamow-Teller strength in medium-mass nuclei) has been studied at TRIUMF in recent years. These and similar experiments addressing questions of nuclear structure are probably among the most important application of the $(n, p)$ reaction, since they address questions that cannot be answered in other ways. This experiment concentrated on two areas: the isovector giant monopole resonance (IVM) and the quasifree continuum. The IVM resonance remains interesting, and its properties have only been observed in the $\left(\pi, \pi^{\circ}\right)$ reaction. [20] Excitation of the quasifree continuum has been the subject of much experimental and theoretical interest. Although the physics questioris may appear disjoint, they are in fact related. The extremely wide momentum acceptance of the MRS $(\Delta p / p= \pm 20 \%)$ mal it possible, even obligatory, to study both regions of excitation simultaneously, arti information on the continuum is important to determine the "background" of the giant resonance region. The analysis of the ${ }^{90} \mathrm{Zr}(n, p)$ data is in progress. This experiment is the basis of a $\mathrm{Ph}$.D. thesis of $\mathrm{H}$. Zhang from Ohio University; more beam time for this work has been requested for the summer of 1993.

LAMPF Experiment No. 881U, Polarization Transfer in Quasifree $(p, n)$ reactions at $500 \mathrm{MeV}$, Ohio University, Indiana University Cyclotron Facility, LANL, and NMSU (Park); T. M. Taddeucci, LANL, Spokesman.

It has long been predicted that the longitudinal channel of quasielastic scattering should be shifted and enhanced with respect to the transverse channel at certain momentum transfers. This effect is due to the momentum-transfer dependence of the particle-hole interaction based on the $\pi+\rho+g$ model.[21] In an earlier experiment (No. 881), polarization transfer measurements were made that isolated the nuclear responses in several nuclei at a momentum transfer of about $1.8 \mathrm{fm}^{-1}$. The surprising result was that no enhancement of the longitudinal channel was seen.

It is important to examine this response at another momentum transfer to help clarify the momentum-transfer dependence of the underlying particle-hole interaction. Therefore, in this experiment we measured a complete set of polarization transfer observables a! $500 \mathrm{MeV}, 12^{\circ}$, corresponding to a momentum transfer of 1.2 $\mathrm{fm}^{-1}$, on ${ }^{2} \mathrm{H},{ }^{12} \mathrm{C}$, and ${ }^{40} \mathrm{Ca}$. Analysis of these data is in progress; the results will be used as part of a Ph.D. thesis of B. Luther.

LAMPF Experiment No. 1040, Spin Transfer Measurements on Quasielastic and Quasifree Delta Production using the $(p, n)$ Reactions at 500 and $800 \mathrm{MeV}$ on 
Several Nuclei, Indiana University Cyclotron Facility, Ohio University, Ohio State University, Niels Bohr Institute, LANL, and NMSU (Park); T. M. Taddeucci, LANL, and C. D. Goodman, IUCF, Spokesmen.

In this experiment we measured both the cross section and the spin depolarization of the $(\mathrm{p}, \mathrm{n})$ reaction over the quasifree delta production region on ${ }^{2} \mathrm{H},{ }^{12} \mathrm{C}$, and ${ }^{40} \mathrm{Ca}$ at $800 \mathrm{MeV}, 0^{\circ}$. Quasifree delta production is the analogue of quasielastic scattering in which the struck nucleon is itself excited. These data compliment the existing data on the delta region using the $\left(p, p^{\prime}\right),\left({ }^{3} \mathrm{He}, t\right)$ and $\left(e, e^{\prime}\right)$ reactions. In addition to this, these data should shed light on the shift in the delta to lower excitations that was larger than was expected, which was noted in both the $\left(e, e^{\prime}\right)$ data and previous $(p, n)$ cross-section data. This effect is thought to be due to pionic correlations in the isovector channel.[22] $D_{n n}$ measurements are predicted to be sensitive to these correlations and therefore should provide valuable information about the nuclear response in the delta excitation region. Analysis of the data is underway at OSU and will be used as a part of Ph.D. requirement for S. DeLucia.

IUCF Experiment No. 344, The ${ }^{10} \mathrm{~B}(p, n){ }^{10} \mathrm{C}$ Reaction at $186 \mathrm{MeV}$, Ohio University, Indiana University, University of Virginia, Ohio State University, LANL, and NMSU (Park); J. Rapaport, Ohio University, Spokesman.

The ${ }^{10} \mathrm{~B}$ nucleus is probably the only stable one in nature for which, to a good approximation, the ground-state wave function can be described as a stretched configuration, i.e., with two nucleons in the $1 p_{3 / 2}$ subshells coupled to a total angular momentum $J^{\pi}=3^{+}$. Thus, scattering and nuclear reactions on ${ }^{10} \mathrm{~B}$ provide a unique opportunity to study transitions between an initial non-spherical target with a very well-described wave function and final states that are more spherical, such as the $J^{\pi}=0^{+}, T=1$ isobaric triplet of mass $A=10$. For this reason, ${ }^{10} \mathrm{~B}$ has been studied with many probes. In particular, the pure $M 3$ electroexcitation of the $T=0$ ground state of to the $T=1, J^{\pi}=0^{+}$level at $1.74 \mathrm{MeV}$ has been studied by Hicks et al.[23] Their results indicate excellent agreement with calculations that employ pure $1 p_{3 / 2}$ single-particle wave functions for the $M 3$ transition.

In this experiment the ${ }^{10} \mathrm{~B}(p, n){ }^{10} \mathrm{C}$ reaction was studied using the Indiana University Cyclotron neutron time-of-flight facilities at an incident proton energy of $186 \mathrm{MeV}$ between $\theta_{\mathrm{lab}}=0^{\circ}$ and $50^{\circ}$ in steps of $5^{\circ}$. The IUCF neutron polarimeter was also used to measure polarization observables at $\theta_{\text {lab }}=0^{\circ}, 15^{\circ}$, and $20^{\circ}$ using an incident transverse-polarized proton beam. Measurements were made to study the $M 3$ stretched ground-state transition, to study the Gamow-Teller (GT) and spin dipole energy distribution, and to obtain structure information to final states in ${ }^{10} \mathrm{C}$. Results for the $M 3$ stretched ground-state transition agree well with cross-section calculations and polarization observables. The distribution of GT strength seems 
to be extended to a much higher energy than the predicted results of $1 p-1 h$ calculations. The same is observed for the spin dipole strength. Resonances at 16.5, 17.3, and $20.3 \mathrm{MeV}$ excitation energy in ${ }^{10} \mathrm{C}$ seem to be the analog of $2^{+}, 2^{-}$and $1^{-}$states previously observed in ${ }^{10} \mathrm{~B}$. This experiment served as a part of $\mathrm{Ph}$. D. requirement of L. Wang of Ohio University, and a paper has been submitted for publication.

LAMPF-WNR Experiment No. 4N0039, A Test of the Effective NN Isovector Tensor Interaction using the Stretched State in the ${ }^{10} B(p, n)^{10} B e(g s)$ Reaction from Bombarding Energies of 70 to $240 \mathrm{MeV}$, University of California (Davis), Ohio University, University of Virginia, Loma Linda Univesity, LANL, and NMSU (Park); D. S. Sorenson, LANL, Spokesman.

This experiment was done in part to complement the work done at IUCF described above. Differential cross sections were measured for úue " $0 \hbar \omega " \mathrm{~J}^{\pi}=0^{+}$ stretched state in the ${ }^{10} \mathrm{~B}(\mathrm{n}, \mathrm{p}){ }^{10} \mathrm{Be}(\mathrm{gs})$ reaction from 70 to $240 \mathrm{MeV}$. The white neutron source at LAMPF.WNR provides neutrons with a continuous energy distribution between 40 and $550 \mathrm{MeV}$. In this energy domain, this is the only facility in the world where the exiergy dependence of the effective nucleon-nucleon interaction can be studied simultaneously over such a range. At momentum transfers between 1 and $2 \mathrm{fm}^{-1}$ the stretched states are mainly excited by the tensor interaction. Using structure information from electron scattering measurements, the Franey-Love interaction has been tested from 90 to $240 \mathrm{MeV}$ and the M3Y interaction has been tested for the $70 \mathrm{MeV}$ data. Analysis of the data is near completion, and a paper is being prepared for publication.

PSI Experiment No. R-87-13, Proposal to Study Multi-particle Final States in Pion-Nuclear Reactions with a Large Acceptance Detector (LADS), Universität Basel, Universität Karlsruhe, LANL, University of Maryland, MIT, Old Dominion University, University of Zagreb, PSI, and NMSU (Kyle, Lin, Wang); Q. Ingram, PSI, Spokesman.

The Large Acceptance Detector System (LADS) at the Paul Scherrer Insitute (PSI) was constructed in order to investigate multiparticle final states resulting from pion interactions in nuclei. The initial experiment, a survey of pion absorption reactions in ${ }^{3,4} \mathrm{He}$ and heavier nuclei at several incident energies, was approved with high priority in the summer of 1987 . The primary interest was to investigate the long-outstanding question of the role of multi-nucleon pion absorption processes and the possibility of exotic mechanisms. $[24,25]$ More generally, the detector was designed to be suitable for investigations of a variety of quasifree reactions, in order to obtain more detailed information about the interactions of the pion, the nucleon, and the delta in nuclei.

The primary detector design goals were a solid-angle acceptance as close to ti:e 
Inaximum $4 \pi$ sr as possible and the capability to reconstruct the full kinematics of the outgoing particles. The design chosen consisted of 196 plastic scintillator counters arranged in a main cylindrical array, which cover most of the solid angle, and two end-cap arrays, which close the ends. Two cylindrical multi-wire chambers with helical cathode strips provide tracking information. The detector covers $98.8 \%$ of the full solid angle. The LADS detector and electronics construction was completed within two years of the proposal at a relatively modest cost of about one million Swiss francs. The NMSU group had primary responsiblity for the design, construction, and operation of the end-cap counters. A limited amount of data were obtained in the fall of 1989.

The PSI accelerator was shut down from January, 1990, until March, 1991. The second run began in May 1991 and continued until mid-October. During the shutdown, work proceeded on the data analysis program, LADYBIRD. Modifications were made to the detector which made the calibration easier and improved background rejection. More flexible trigger electronics were introduced. Addition of a CERN Host Interface (CHI) in FASTBUS increased data taking rates a factor of eight to about $400 \mathrm{~Hz}$. Several computer tasks monitored the phototube gains and high voltages, beamline magnets, event scalers, and raw detector spectra. A VAX workstation provided by NMSU performed some of these tasks.

In 1991, data were taken on pressurized gas targets of ${ }^{2} \mathrm{H},{ }^{3,4} \mathrm{He},{ }^{14} \mathrm{~N}$, and ${ }^{40} \mathrm{Ar}$ at incident pion momentz of 220,270 , and $355 \mathrm{MeV} / \mathrm{c}$. The logic permitted several different trigger types according to charged and neutral particle multiplicities, which were prescaled to emphasize events with two or more charged particles. Typically, 20 million events were taken for each target, energy setting in about one day of running. For the ${ }^{4} \mathrm{He}$ data, 40 millioa events were taken per setting. About 400 gigabytes of data were taped.

Tests were also made of a neutron time-of-flight detector wall in coincidence with the LADS. Previous studies suggested that multi-nucleon absorption processes often involve emission of one or more energetic neutrons.[26, 27] The LADS detector has good neutron detection efficiency but poor neutron energy resolution, and therefore it was designed to run at high beam inte nsities in coincidence with external detectors. The test results indicate that such rur.ning is feasable.

In the spring of 1993 we plan a third run to complete the initial survey experiment which was proposed to measure pion absorption reactions on six targets, ${ }^{2} \mathrm{H},{ }^{3}, 4 \mathrm{He}$, ${ }^{14} \mathrm{~N},{ }^{40} \mathrm{Ar}$, and $\mathrm{Xe}$ at five pion energies. During the past year several important improvements have been made to the detector system. The inner wire chamber has been redesigned to incorporate two independent chambers which better determine the track reconstruction efficiency. Data taking rates have been further improved to about $1000 \mathrm{~Hz}$ due to several changes in the electronics and computer system. The 
relatively slow LeCroy $2800 \mathrm{ADC}$ 's for the wire chamber cathode strips have been replaced by 1882 Fastbus ADC's. The data is passed from the CHI to a faster VAXstation 4000 Model 90 (contributed by NMSU) by a DRQ to Turbochannel interface in VME. The use of 5 gigabyte Exabyte tape drives increases the recording speed. The higher data rates are important both for lessened systematic uncertainties, because the data is taken more quickly and with smaller deadtime corrections, and for better statistical precison when studying kinematic distributions in restricted regions of phase space.

The technical effort at NMSU, together with the group from Old Dominion University (ODU), has primarily focussed improving the Monte Carlo program, which is based upon the GEANT and GHEISHA libraries from CERN. Better event generators using the plane wave impulse approximation (PWIA) with realistic spectral functions, derived from electron scattering information when possible, have been written. It is now possible to write Monte Carlo data to a pseudodata file which can be analyzed with LADYBIRD using the same cuts as for the LADS data. Work is in progress on better simulations of hadronic interactions in the detector in order to better determine their effect on measured kinematic distributions and on the detector efficiency for charged and neutral particles.

Our analysis effort has concentrated on the $355 \mathrm{MeV} / \mathrm{c}$ data. This work is being done together with the ODU group and W. R. Gibbs, a theoretician at NMSU. LADS was built to study the mechanisms responsible for pion absorption reactions leading to multinucleon final states. It is now well established that only about half of the total absorption in ${ }^{4} \mathrm{He},{ }^{16} \mathrm{O}$, and ${ }^{58} \mathrm{Ni}$ above $165 \mathrm{MeV}$ can be explained by the observed two nucleon absorption yield including an estimate of final state interactions. $[26,28,29]$

One open question is the role of initial state interactions (ISI), where an essentially on-shell pion removes one or more nucleons prior to absorption on two nucleons. It is known that multiple pion scattering contributes about $15-20 \%$ to the total inelastic scattering in ${ }^{16} \mathrm{O}$ at $165 \mathrm{MeV},[30]$ and there is evidence that the process is largely on shell.[31] A model of Masutani and Yazaki,[32] which includes only on-shell pion propagators and free $\pi \mathrm{N}$ amplitudes, is able to fit the total absorption and inelastic scattering cross sections. The model calculates multiple scattering multiplicities which are consistent with the inelastic data and are predicted to be large for pion absorption above about $115 \mathrm{MeV}$. However, the experimental evidence for ISI in pion absorption is weak. While some experiments have observed peaks in the forward proton energy distributions near the energy of free $\pi$-p scattering, $[33,34]$ a signature of ISI, the yield is rather small and insufficient to explain the total absorption cross sections. Also, the observation of considerable strength in the longitudinal response for electron scattering in the dip region may reflect a 
new multinucleon response to absorption of a virtual photon that can contribute to pion absorption as well. One goal of the LADS analysis is to distinguish these mechanisms.

Studies are underway at NMSU using the Intranuclear Cascade Code (INC) developed by Gibbs to identify kinematic signatures of ISI and FSI and possibly determine their strength. The INC models the effects of Fermi motion, binding, and geometry, but it contains no explicit mutinucleon absorption process. Therefore the ${ }^{4} \mathrm{He}\left(\pi^{+} 3 \mathrm{p}\right) \mathrm{n}$ reaction can proceed either by an on-shell $\pi \mathrm{N}$ scattering followed by absorption on two nucleons (ISI), or by a two-nucleon absorption followed by an NN scattering (FSI). One quantity of interest is the dot-product $P_{i j} \equiv \vec{P}_{i} \cdot \vec{P}_{j}=$ $P_{i} P_{j} \cos \left(\theta_{i j}\right)$ taken for all proton pairs $(\mathrm{i}, \mathrm{j})$. Naively FSI is expected to produce a peaking at zero due to the $90^{\circ}$ opening angle for on-shell NN scattering. In contrast, the absorbing pair ("right pair") after ISI should have a larger opening angle, giving large negative $P_{i j}$ values similar to those for two-nucleon absorption without ISI. This opening angle is rather insensitive to the absorbed pion energy. These features are apparent in Fig. 4, which shows the LADS data (upper left), Monte Carlo phase space (upper right), INC for ISI events (lower left), and INC for FSI events (lower right). The INC distributions (which include both "right" and "wrong" pairs) show the expected structures broadened by Fermi motion and binding. The LADS data appear to show corresponding structures and are very different from the phase space distributions.

PSI Experiment R91-11, Cross Sections and Anaiyzing Power Measurements for ${ }^{7} \mathrm{Li}(\pi, \pi p)^{6} \mathrm{He}$ with Good Kinematic Resolution, Old Dominion University, University of Maryland, Universität Karlsruhe, and NMSU (Kyle, Wang, Lin, Chang); N. Chant, Maryland, A. Klein, Old Dominion, and G. Kyle, Spokesmen.

This experiment, which was originally proposed as LAMPF Experiment E1172, was not run there due to the low ${ }^{7} \mathrm{Li}$ polarization obtained with the target material and a ${ }^{3} \mathrm{He}$ cryostat. Because a suitable ${ }^{3,4} \mathrm{He}$ dilution cryostat was not available at LAMPF, the proposal was submitted to PSI in January, 1992, and ran for eleven weeks starting in July, 1992.

We measur . crc is sections and analyzing powers for the reaction ${ }^{7} \operatorname{Li}\left(\pi^{+}, \pi^{+} p\right)^{6} \mathrm{He}$. The scattered pions were analyzed with the SUSI spectrometer, and the proton energies were measured with an array of plastic scintillation counters which we designed and helped construct. Measurements were made at $240 \mathrm{MeV}$ at pion angles of $60^{\circ}, 80^{\circ}$, and $110^{\circ}$, with coincident proton angles roughly centered about the free kinematics region. The ${ }^{7} \mathrm{Li}$ target polarization was typically $35-45 \%$.

The aim of this experiment is to study the two different types of asymmetries that arise from pion-proton quasielastic scattering. One of these is the intrinsic 


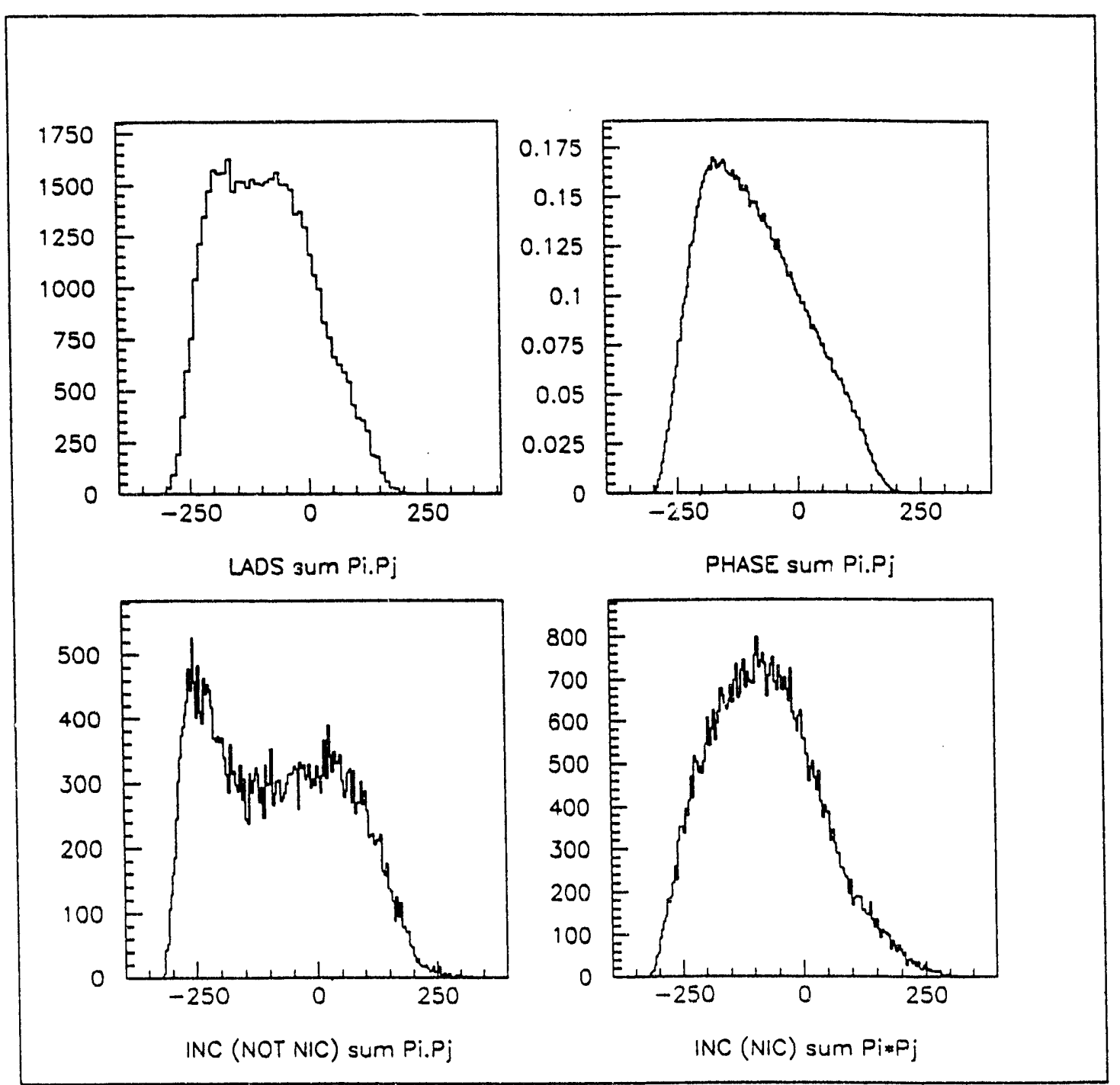

FIG. 4. Comparison of LADS data with Monte Carlo phase space and the Intranuclear Cascade Model, from PSI Exp. R-87-13. The histrograms are described in the text. 
pion-nucleon asymmetry, as modified by the nuclear medium. The other arises from the relatively strong optical absorption of the pion, compared to the proton, which localizes the the reaction on one side of the polarized nucleus. This is known at Newn's polarization, and it has been discussed in the context of pion-nucleus scattering by Gibbs and Siegel.[35] According to calculations of Roos and Chant,[36] it should be possible to separate these effects in an experiment of this kind, since forward scattering should contain strong contributions from the spin dependence of $\pi p$ scattering and backward scattering relatively little. These results may help shed light on the unexpectedly small asymmetries observed in elastic scattering on ${ }^{13} \mathrm{C}$ and ${ }^{15} \mathrm{~N}$, and they may also be sensitive to the strength of the delta-nucleus spin-orbit interaction. The data analysis is underway at Maryland as part of the Ph.D. thesis of Mohammed Khayat.

An update (Experiment R91-11.2) to study the pion absorption reaction ${ }^{7} \mathrm{Li}\left(\pi^{+}, \mathrm{pp}\right)$ in a polarized target was approved in December, 1992, and is scheduled to be run in summer, 1993, using the same apparatus. The motivation is to study medium modifications of the quasideuteron absorption process and the effects of distortions. This experiment will be the basis of a Ph.D. thesis for Tom Greco, a student at Old Dominion University. 


\subsection{Analysis of Past Experiments}

Work has been in progress on the analysis of data and the interpretation of the results of past experiments, in preparation for publication. This work includes the following:

LAMPF Experiment No. 316, M asurements of Low-Energy $\pi^{-}-N u c l e u s$ Elastic Scattering, Northwestern University, MIT, Saclay, LANL, and NMSU; K. K. Seth, Northwestern, and G. Burleson, Spokesmen.

In this experiment, run several years ago, we measured cross sections for $\pi^{-}$ scattering on a number of nuclei, from ${ }^{12} \mathrm{C}$ to ${ }^{208} \mathrm{~Pb}$, at $20,30,40$, and $50 \mathrm{MeV}$, as well as some $\pi^{+}$scattering at $50 \mathrm{MeV}$, using a range telescope of scintillation counters. The 30 - and $50-\mathrm{MeV}$ results have been published,[37] but the remainder of the data have not, partly because there were no reliable normalizations. During summer, 1992, measurements were carried out on the Clamshell spectrometer in the LEP channel at LAMPF which obtained data to give a normalization at $40 \mathrm{MeV}$. With that, together with normalizations of data similar to ours at $20 \mathrm{MeV}$, which also came from Clamshell measurements, we plan to submit a final paper on these results. The $20-\mathrm{MeV}$ data are shown in Fig. 5 , together with predictions of the MSU model.[38] As was found previously with a similar model,[37] the agreement is good for the smaller nuclei but poor for the heavier nuclei.

PSI Experiment No. R-83-28, A Measurement of the Reaction ${ }^{16} O\left(\pi^{+}, p p\right)^{14} \mathrm{~N}$ over a Broad Kinematic Range for Pion Energies of 115 and $169 \mathrm{MeV}$, University of Maryland, MIT, PSI, and NMSU (Kyle); G. Kyle, Spokesman.

This experiment, which was run in 1986-87, studied the role of the simplest quasifree absorption process in which a pion is absorbed on a nucleon pair in ${ }^{16} \mathrm{O}$. Previous inclusive measurements of ${ }^{12} \mathrm{C}\left(\pi^{+}, \mathrm{pp}\right)$ by Altmann suggested that the unperturbed two-nucleon process constituted only about $10 \%$ of the total absorption at $\Delta(1232)$ resonance energies. [40] Such a small contribution is difficult to reconcile with DWIA models, which successfully explain inclusive $\left(\pi^{+}, \mathrm{p}\right)$ data, and with more recent exclusive measurements of ${ }^{58} \mathrm{Ni}\left(\pi^{+}, \mathrm{pp}\right) \cdot[29,41,42] \mathrm{In}$ order to resolve these discrepancies, we measured the exclusive $\left(\pi^{+}, \mathrm{pp}\right)$ reaction which made possible a better separation of the two-nucleon absorption from the multinucleon "background" and also gave information about the contributions from nucleons in different shells. The data were analyzed by two Ph.D. students, David Mack and Scott Hyman, at Maryland.[43, 44]

After correction for final state interactions, the data show that a two-nucleon process can explain about $75 \%$ of the total absorption cross section at $115 \mathrm{MeV}$. A substantial contribution from cross-shell absorption on s- and p-shell nucleon 


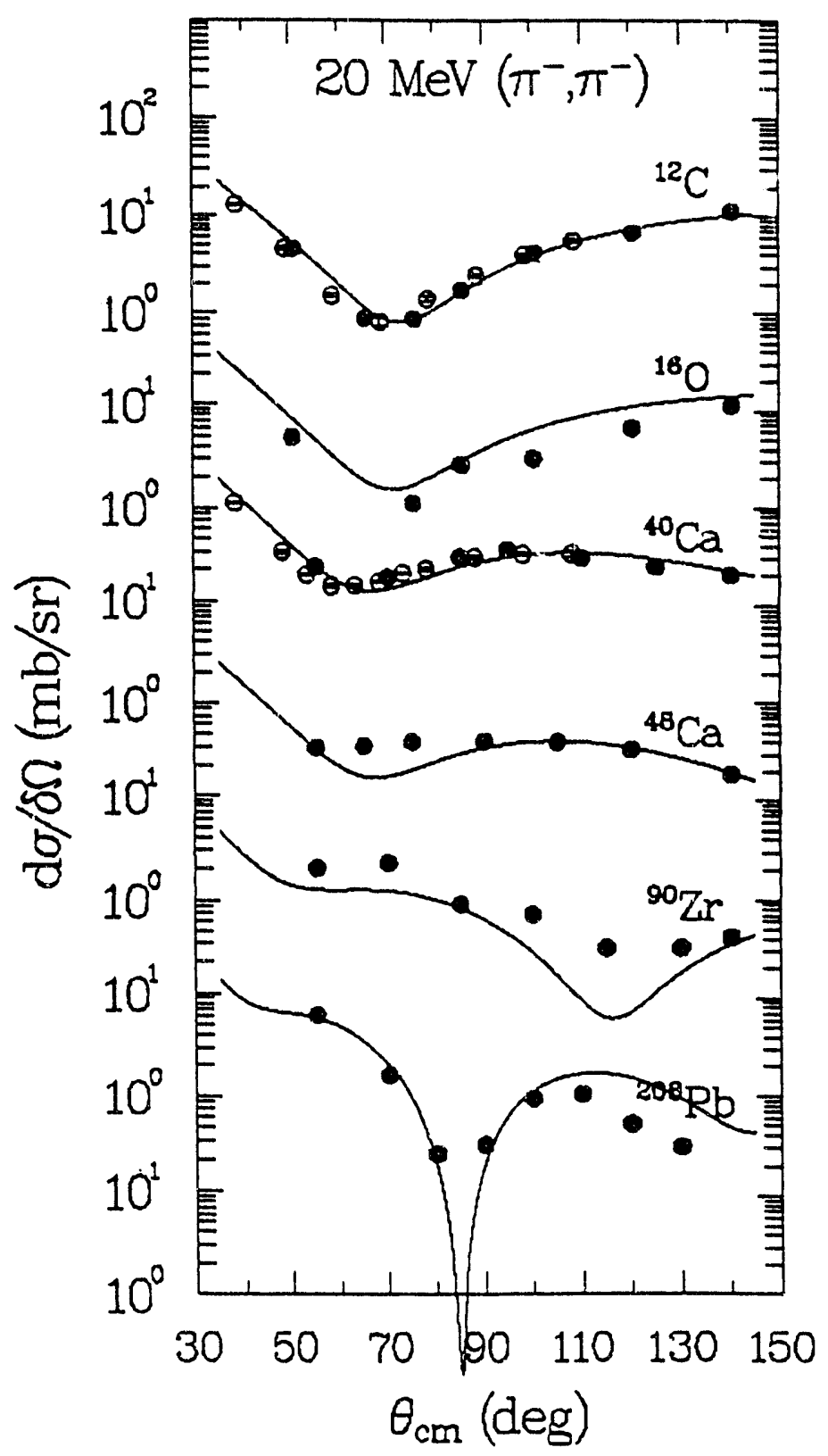

FIG. 5. Differential cross section for $\pi^{-}$elastic scattering from the nuclei indicated, from LAMPF Exp. 316, compared with previous measurements.[39] The curves are predictions of the MSU model.[38] 
pairs was seen. At $165 \mathrm{MeV}$, the fraction dropped to about $40 \%$. The uncorrected two-nucleon cross section was about twice that reported by Altmann. A similar discrepancy was seen by Burger in the case of ${ }^{58} \mathrm{Ni}$.[29] The disagreement apparently arises from their cross section normalization rather than from energy thresholds. If we apply similar acceptance cuts to our data the angular correlations have similar shape but also have about twice the magnitude of Altmann's. Therefore it appears that the Altmann experiment seriously under-reports the strength of two-nucleon absorption.

A brief report on the $165 \mathrm{MeV}$ results and a paper describing the $115 \mathrm{MeV}$ results have been published. $[28,45] \mathrm{A}$ more complete paper on the comparison of the two energies in preparation.

PSI Experiment No. R-87-05.1, A Study of the Reaction ${ }^{6} \mathrm{Li}\left(\pi^{+}, p p\right)$ over the $\Delta$ (1292) Resonance Region, Arizona State University, University of Maryland, PSI, and NMSU (Kyle, Dhuga, Wang); P.G. Roos, Maryland, and G. Kyle, Spokesmen; and LAMPF Experiment No. 948, Quasideuteron Absorption on ${ }^{6} \mathrm{Li}$ and ${ }^{10} \mathrm{~B}$, Arizona State University, University of Maryland, University of South Carolina, and NMSU (Kyle, Rawool); B. G. Ritchie, ASU, Spokesman.

These proposals studied the two-nucleon absorption process in ${ }^{6} \mathrm{Li}$ for eight energies between 30 and $220 \mathrm{MeV}$. The purposes were to measure the A-dependence of the two-nucleon process, and to corpare the absorption on the loosely-bound valence nucleons with that on the tightly-bound core nucleons. The LAMPF experiment was run in December, 1986, and the PSI experiment followed in summer, 1987. Details are described in our previons progress reports. Ph.D. students Dahai Zhang at Maryland (PSI) and Dennis Rothenburger Arizona State (LAMPF) analyzed these data as a part of their dissertations, [46] and publications are in preparation.

PSI Experiment No. R-87-09, Pion Absorption in Flight and Nucleon Multiplicities, NIKHEF, University of Maryland, NIKHEF-K (Amsterdam), KFK Karlsruhe, PSI, and NMSU (Kyle, Mukhopadhyay, Wang); H. Breuer, Maryland, and Th.S. Bauer, NIKHEF, Spokesmen.

This experiment, which was run at PSI in 1988, studied the absorption of pions in ${ }^{4} \mathrm{He}$ and ${ }^{16} \mathrm{O}$ at energies around the $\Delta(1232)$ resonance, using a detector array which covered about $55 \%$ of the full solid angle. The motivation was to study the A-dependence of the absorption cross section in light nuclei. Previous measurements indicate that the total absorption cross section increases about a factor of four from ${ }^{3} \mathrm{He}$ to ${ }^{4} \mathrm{He}$, out of proportion to the increase in nucleon pairs, while the inelastic cross section showed a corresponding drop. $[47,48]$ This behavior might indicate the onset of a strong four-nucleon absorption mode, which could possibly arise from the very interesting $\Delta N \rightarrow \Delta \Delta$ process. $[24,25]$ 
The reactions ${ }^{4} \mathrm{He}\left(\pi^{+}, 2 \mathrm{p}\right)$ and ${ }^{4} \mathrm{He}\left(\pi^{+}, 3 \mathrm{p}\right)$ were measured at several energies from 65 to $330 \mathrm{MeV}$, and the previous NIKHEF measurements on ${ }^{16} \mathrm{O}$ were extended to 115 and $165 \mathrm{MeV}$.[49,50] The data analysis was based upon a Monte-Carlo simulation of the experiment using quasi-free phase space events generators. First results, which have been published, show that, after correction for effects of final state interactions, the ${ }^{4} \mathrm{He}\left(\pi^{+}, 2 \mathrm{p}\right) \mathrm{pn}$ reaction can explain only about $50 \%$ of the total absorption cross section.[51] Analysis of the 3p channel is underway at Maryland as part of the Ph.D. thesis of Farida Adimi. 


\subsection{Preparation for Future Experiments}

Work has also been in progress on plans and preparations for future experiments, including submissions of proposals to laboratory advisory committees. This includes the following:

LAMPF Experiment No. 1256, $\pi^{ \pm} \vec{p}$ Analyzing Powers at 45 and $67 \mathrm{MeV}$, Arizona State University, University of Minnesota, Abilene Christian University, Rudjer Boskvic Instutite, Old Dominion University, University of Texas, University of Wyoming, LAMPF, and NMSU; J. R. Comfort, Arizona, and G. Burleson, Spokesmen.

The physics of this proposed measurement involves studies of the mechanism of the breaking of chiral symmetry, which is generally believed to be related to the interaction responsible for producing non-zero quark masses. A test of this is the evaluation of the so-called "sigma" term.[52] Values for this quantity can be found both from the formalism related to the model of hadron mass splitting and from analysis of low-energy pion-nucleon scattering data. Naive estimates of the former give a value of about $25 \mathrm{MeV}$, while analyses of pion-nucleon data have given values of $64 \pm 8 \mathrm{MeV}$. Part of the discrepancy is believed to stem from the strange-quark content of the nucleon, to which the sigma term is related. A precise evaluation of this quantity would give information on the fraction of strange quarks in the nucleon.

The problem in evaluating the sigma term from experimental pion-nucleon data is that there are major unresolved discrepancies among the various existing lowenergy data sets, all of which consist of differential cross-section measurements. It is the purpose of this experiment to try to resolve some of these discrepancies by making measurements of a new quantity, the asymmetry of $\pi^{ \pm}$scattering from a polarized proton target. We plan to take data at 45 and $67 \mathrm{MeV}$, where differential cross-section measurements exist.

The experimental setup used will be essentially the same as used previously by this group.[19] Some modifications will be necessary, because of the low energy of the pions. These include the use of a dilution refrigerator with a thin window rather than a ${ }^{3} \mathrm{He}$ cryostat, to allow running in frozen-spin mode with low magnetic fields around the polarized target, and providing for the rotation of the refrigerator so as to minimize the contribution of the target thickness to the experimental resolution. During the summer, detailed plans were made for the preparations necessary to run this experiment in summer, 1993, with task assignments for the experimenters and the laboratory personnel involved. This work is currently in progress. This experiment is expected to be the basis of a Ph.D. thesis for Christopher Allgower of Arizona State University. 
LAMPF Experiment No. 1267, Elastic Scattering of $\pi^{+}$and $\pi^{-}$from Polarized ${ }^{3} \mathrm{He}$ at $T_{\pi}=180 \mathrm{MeV}$ to $475 \mathrm{MeV}$, University of Minnesota, TRIUMF, Ohio University, Tel Aviv University, LANL, and NMSU; D. Dehnhard, Minnesota, O. Häusser, TRIUMF, and G. Burleson, Spokesmen.

For this work, we plan to bring a polarized ${ }^{3} \mathrm{He}$ target from TRIUMF to LAMPF. This target has been used for several experiments at TRIUMF $[53,54,55]$, and it has been described in the literature.[56] At LAMPF, we plan to measure the analyzing power $A_{y}$ for both $\pi^{ \pm}$elastic scattering from polarized ${ }^{3} \mathrm{He}$ at incident energies between 180 and $475 \mathrm{MeV}$. Such measurements are not easily feasible at TRIUMF because of the lower beam rates there and the absence of suitable spectrometers. At LAMPF, such measurements are easily practical.

Recent measurements of $A_{y}$ for pion scattering from ${ }^{3} \mathrm{He}$ at $100 \mathrm{MeV}$ found large values,[55] but similar measurements with ${ }^{13} \mathrm{C}$ and ${ }^{15} \mathrm{~N}$ found values of $A_{y}$ small or consistent with zero, $[19,57]$ in contrast to the large values predicted by theory. $[58$, 59] For the p-shell nuclei, the predicted asymmetries showed a strong dependence on the details of the nuclear structure, which suggests that information on nuclear spin excitations is contained in the data. The failure of the calculations to reproduce the measured values of $A_{y}$ implies that the $\pi$-nucleus reaction mechanism, as related in particular to spin-flip scz'tering, is not sufficiently understood. This suggests the importance of measuring asymmetries on a nucleus of well-known structure, for which the effects of the reaction mechanism and the nuclear structure can be separated.

Such a nucleus is ${ }^{3} \mathrm{He}$, since reliable wave functions have been found for it by Faddeev calculations. $[60,61,62]$ The $100-\mathrm{MeV}$ pion asymmetry data were reproduced fairly well by optical model calculations,[55] as well as by a simple model using only free pi-nucleon amplitudes with a simple ${ }^{3} \mathrm{He}$ form factor.[63] At higher energies, however, predictions of this model and of a full multiple-scattering theory diverge,[63] leading to a prediction of a sign change of $A_{y}$ at an energy of about $215 \mathrm{MeV}$. The predictions are also different for $\pi^{+}$and $\pi^{-}$. In this experiment, we propose to measure this asymmetry at five, energies from 180 to $475 \mathrm{MeV}$, at angles where the maximum effects are expected. From this we hope to find information which can be used to help understand the nature of pion spin-dependent interactions in nuclei, and of spin-dependent nuclear structure as well.

During the summer, beam tests were carried out at the $\mathrm{P}^{3}$ channel at LAMPF, using the Large Aperture Spectrometer (LAS) with a target brought from TRIUMF that is similar in geometry to the polarized target. The aim was to develop a system, using collimators and anticoincidence counters, that would prevent scattering from the glass walls of the target. This test was successful, we are convinced that the experiment can be performed in this channel, and we expect that it will be scheduled 
in summer, 1993. This experiment should be the basis of a $\mathrm{Ph} . \mathrm{D}$. thesis for Michelle Espy, of the University of Minnesota.

LAMPF Proposal No. 1269, Precise Measurements of Isovector Transitions in ${ }^{13} \mathrm{C}$ and ${ }^{7} \mathrm{Li}$ induced by Pion Single Charge Exchange, University of Colorado, MIT, University of Pennsylvania, Arizona State University, LA $: J L$, and NMSU; E. R. Kinney, University of Colorado, and G. Burleson, Spokesmen.

At meetings of the NMS collaboration group in summer and fall, 1991, it was decided to propose this experiment as part of a first-round physics program with the NMS, since it was felt that the other proposals existing at that time appeared somehow to be too technically demanding to be sensibly attempted as first experiments. Two other experiments were recommended as well, to be run in 1992, when the NMS was not in its final configuration; one of these was Exp. No. 1268, described above. It was felt that ${ }^{13} \mathrm{C}$ was a particularly good target for this purpose, because this nucleus has a range of well-separated excited states in SCX transitions (both single-particle and collective), because the counting rates are high, and because the targets can be fabricated so as to allow for the variation of volume density needed to achieve maximum resolution. The physics involved in these studies is of great interest as well. When this proposal was presented to the LAMPF Program Advisory Committee (PAC), it was felt that the ${ }^{7} \mathrm{Li}$ work was of less interest, so we are not currently planning to take data with that target.

In this experiment we propose to measure angular distributions for $\left(\pi^{+}, \pi^{0}\right)$ and $\left(\pi^{-}, \pi^{+}\right)$on ${ }^{13} \mathrm{C}$ for both ground states and excited states, at 162 and $292 \mathrm{MeV}$. Previous studies $[64,65]$ of $\left(\pi^{+}, \pi^{-}\right)$were unable to cleanly resolve the ground state from the first excited state of the recoiling nucleus. These data, together with previous measurements[66] of elastic and inelastic scattering of $\pi^{ \pm}$from ${ }^{13} \mathrm{C}$, will allow, for the first time, an isospin decomposition of the transition amplitudes for pion scattering to these states. This includes extraction of $\mathrm{T}=1 / 2$ and $\mathrm{T}=3 / 2$ amplitudes, as well as isoscalar and isovector amplitudes, with their relative phase angles. Examples of such extractions, using existing data, were given in the proposal. In addition, the reaction ${ }^{13} \mathrm{C}\left(\pi^{-}, \pi^{0}\right){ }^{13} \mathrm{~B}$ will be investigated to cleanly study transitions to $\mathrm{T}=3 / 2$ states (and possibly $\mathrm{T}=5 / 2$ states). The results of theoretical analyses of these data are expected to be sensitive to microscopic details of the pion-nucleus interaction, expectially the effects of direct $\Delta$-nucleon interactions.

We also propose to investigate a predicted[67] energy variation of the position of the minimum of the ground-state isobaric analog transition which is similar to that seen in analaog transitions in DCX scattering. After many years of investigation, an anomalous shift in the minimum of DCX analog transitions near the peak of the $\Delta$ resonance has not been satisfactorily explained. It is speculated that the proposed 
measurement may indicate that the anomaly can be attrributed to larger radii of the valence neutrons than is cuttently believed.[68]

LAMPF Proposal No. 1300, Measurement of Asymmetries $A_{y}$ and Differential Cross Sections of Pion-Induced Single Charge Exchange on Polarized ${ }^{3} \mathrm{He}$, University of Minnesota, TRIUMF, Simon-Fraser University, Rutgers, Ohio University, LANL, and NMSU; D. Dehnhard, Minnesota, O. Häusser, Simon-Fraser, and B. K. Park, Spokesmen.

A proposal has been submitted to measure the analyzing powers $A_{y}$ and differential cross sections for the ${ }^{3} \mathrm{He}\left(\pi^{-}, \pi^{\circ}\right)^{3} \mathrm{H}$ reaction at $\mathrm{T}_{\pi}=300$ and $400 \mathrm{MeV}$ between $80^{\circ}$ and $120^{\circ}$. The measurements are to be done with the Neutral Meson Spectrometer (NMS) in an energy region where the $\pi$-nucleus interaction is strongly energy- and isospin-dependent. The pion-induced single-charge-exchange (SCX) reaction on ${ }^{3} \mathrm{He}$ principally involves only one proton in the nucleus, the one with its spin direction opposed to that of the neutron which carries most of the nuclear spin. Thus measurements of $A_{y}$ in SCX allow studies of the isovector spin-dependent part of the pion-nucleus interaction and the tri-nucleon spin density.

IUCF Proposal No. 92-105, Cross Section and $D_{N N}(\theta)$ Measurements in ${ }_{16,17,18} \mathrm{O}(p, n)$ Reactions at 100 and $186 \mathrm{MeV}$, University of Minnesota, IUCF, Ohio University, Ohio State University, LANL, and NMSU; M. Palarczyk, Minnesota, C. D. Goodman, IUCF, and B. K. Park, Spokesmen.

This proposal was approved in December, 1992, and the experiment is likely to be done in fall, 1993. We will measure differential cross sections for the ${ }^{16,17,18} \mathrm{O}(\mathrm{p}, \mathrm{n})$ reactions at 100 and $186 \mathrm{MeV}$ incident proton energies, to decompose the $L=1$ resonances into non-spin-transfer and spin-transfer transitions. The GT strength and the $\mathrm{L}=1$ resonances in ${ }^{16,17,18} \mathrm{~F}$ will be determined up to about $30 \mathrm{MeV}$ excitation energy using a multipole decomposition analysis. It is essential to understand the behavior of the $\mathrm{L}=1$ resonances to correctly identify the GT strength at higher excitation energies and to have a good model for the continuum background. We also will measure the spin-transfer coefficient $D_{N N}\left(0^{\circ}\right)$ for the ${ }^{17} \mathrm{O}(\vec{p}, \vec{n}){ }^{17} \mathrm{~F}($ g.s. $)$ and ${ }^{18} \mathrm{O}(\vec{p}, \vec{n})^{18} \mathrm{~F}(1.04 \mathrm{MeV})$ reactions. This experiment will study anomalies in the behavior of spin-transfer coefficients and cross sections for mirror target transitions, i.e. mixed GT and Fermi transitions, for targets with $\mathrm{J}>0$.

TRIUMF Proposal No. 623, Kinematically Complete Meaurements of Quasifree Pion-Nucleus Single and Double Scattering Reactions, University of Maryland, Old Dominion University, University of British Columbia, TRIUMF, and NMSU; G. Smith, TRIUMF, G. Kyle, Spokesmen.

This proposal was approved in December, 1990, to use the CHAOS detector, 
a magnetic spectrometer with $360^{\circ}$ angular coverage which is under construction at TRIUMF. We will initially measure the exclusive $\left(\pi^{ \pm}, \pi^{ \pm} p\right),\left(\pi^{ \pm}, \pi^{ \pm} 2 p\right)$, and $\left(\pi^{+}, \pi^{-} 2 p\right)$ reactions in ${ }^{4} \mathrm{He}$ at 165 and $240 \mathrm{MeV}$. Later, measurements may be extended to other targets and energies.

One goal is to study the competition of different reaction mechanisms and ultimately gain new information about the delta-nucleon interaction. In the delta-hole model, two types of amplitudes are expected to contribute to nucleon removal: the direct (sequential) removal involving formation and decay of the $\Delta(1232)$-resonance, and nucleon removal involving hard $\Delta-N$ scattering. [69] Previous experiments have observed large variations in the cross section ratios for $\left(\pi^{+}, \pi^{+} p\right) /\left(\pi^{-}, \pi^{-} p\right)$, which cannot be explained with the direct amplitude alone but were explained by the delta-hole predictions.[14, 70,71] From these models the strength of the $\Delta-N$ interaction in isospin, $I=1,2$, channels was obtained. $[15,72]$ A similar interaction was required to explain measurements of the polarization asymmetry, iT 11 , for $\pi^{+} d$ elastic scattering. [73]

Measurement of the double scattering and double charge exchange reactions will further test this model. The double scattering reaction $\left(\pi^{+}, \pi^{+} 2 p\right)$ should be dominated by the sequential formation and decay of the delta, whereas the $\left(\pi^{+}, \pi^{-} 2 p\right)$ and $\left(\pi^{-}, \pi^{-} 2 p\right)$ reactions should be strongly modified by the $\Delta-N$ scattering amplitude, which gives rise to the DINT diagram mentioned above for coherent DCX.

This experiment will also measure the multiplicities of emitted nucleons, which are related to the mean-free paths for pion and nucleon propagation in nuclei, and test theoretical models of the reactive content of the pion-nucleus optical potential.[32] The roles of Initial State Interaction (ISI) and Final State Interaction (FSI) in quasifree reactions, which is an important issue for understanding multinucleon pion absorption, would also be studied.

Construction of CHAOS detector has been completed and commissioning is in progress. First experiments are planned for summer, 1993. We expect this experiment to run in early 1994 and to be a Ph.D. project for an NMSU student.

HERMES: A Proposal to Measure the Deep Inelastic Spin-Dependent Structure Functions of the Nucleon at HERA, Argonne National Laboratory, Caltech, University of Colorado, DESY, University of Erlangen-Nürnberg, LNF Frascatti, MPI Heidelberg, University of Illinois, University of Liverpool, NIKHEF, University of Marburg, MIT, Universität München, INFN Rome, St. Petersburg Nuclear Physics Institute, Universita di Torino, TRIUMF/Alberta/Simon Fraser, University of Wisconsin, Yerevan Physics Institute, IfH DESY-Zeuthen, and NMSU; R. Milner, MIT, and K. Rith, Heidelberg, Spokesmen.

Deep inelastic electron scattering has played a crucial role in the development 
of quantum chromodynamics (QCD). Within the parton picture, the deep inelastic structure functions provide information about the momentum distributions of quarks and gluons in the nucleons. The spin-dependent structure functions, measured using polarized beam and target, provide information on the spin distribution of partons in the nucleon.[74] Perturbative QCD makes definite predictions about the evolution of the structure functions with momentum transfer $q^{2}$ and their shape at large $x$ (the parton momentum fraction). $[75,76,77]$ The dependence of the structure functions on $x$ provides a constraint on hadron models, which contain ideas about the nonperturbative aspects of QCD and yield vastly different predictions for the spin-dependent structure functions of the nucleons. Of particular interest are the Bjorken[78] and Ellis-Jaffe[79] sum rules involving integrals of the spin structure functions. The Bjorken sum rule, which has not been experimentally tested, depends upon only very general properties such as current algebra. The Ellis-Jaffe sum rule, which seems to be violated by the experimental results on the proton, requires additional assumptions such as the absence of sea quark polarization.

Only two measurements, with rather large uncertainties, have been made of the spin-dependent structure function $g_{1}(x)$ for the proton, $[80,81]$ and none have been made for the neutron to date. The results of these measurents have indicated the surprising result that the spin of the proton is not related very strongly to the spin of its quarks. This clearly should be investigated further. Experiments in progress at CERN and SLAC may further clarify this situation.

The HERMES collaboration has proposed to measure the spin-dependent structure functions of the proton and neutron with a precision unmatched by other techniques, using the $30-\mathrm{GeV}$ electron beam of the HERA accelerator at the DESY laboratory in Hamburg, Germany. The experiment involves scattering polarized electrons from polarized gas targets of hydrogen, deuterium and ${ }^{3} \mathrm{He}$. The scattering asymmetries $A_{1}(x)$ and $A_{2}(x)$ can be measured with much higher precision than previously because of the high current $(60 \mathrm{~mA})$ and polarization $(60 \%)$ expected for the beam and the low dilution of polarized nucleons in the gas targets. A unique feature of the HERMES detector is the ability to tag leadling pions and possibly kaons which can help separate the contributions of valence and sea quarks. The possibility to measure the azimuthal distributions of leading hadrons will help to separate contributions of quark spin and orbital angular momentum to the nucleon spin. The experiment will also set upper limits for two deuteron structure functions, $b_{1}(x)$ and $\Delta(x)$, which are expected to be small but which in principal can contribute to the deep inelastic cross section for a polarized deuteron target.

The detector system will consist of a magnetic volume with tracking multi-wire proportional chambers for primary energy measurement, a transition radiation detector for hadron rejection, a Cerenkov counter for $\pi / K$ identification, a trigger 
hodoscope, and a calorimeter for background rejection which also serves as the firstlevel trigger. NMSU has taken primary responsibility for implementation of the first-level trigger and overall responsibility for the first and second level triggers. We are also working together with Caltech, Illinois, NIKHEF, Frascati and Yerevan on the calorimeter design and construction. $\mathrm{O} . \mathrm{s}$ of our students would also analyze a portion of the data.

The proposal for this experiment was presented to the Physics Research Committee (PRC) at DESY in March and September, 1990, and full approval was recommended in September, 1992, after the technical feasablility was demonstrated. During the past year it was demonstrated that a target thickness and polarization of $\mathrm{P}^{2} \rho>10^{14} / \mathrm{cm}^{2}$ can been achieved for the ${ }^{1} \mathrm{H}$ and ${ }^{2} \mathrm{H}$ atomic beam source, and $56 \%$ transverse electron beam polarization has been sustained in the HERA ring. A funding proposal for the U.S. participation is in preparation. It will be submitted to the DOE and NSF in January, 1993. Most of the Canadian and European funding for the project is approved, and design work is proceeding rapidly, with the aim to mount the experiment in winter, 1995. 


\section{References}

[1] A. L. Williams, et al, Phys. Lett. B216, 11 (1989).

[2] A. L. Williams, et al, Phys. Rev. C 43, 766 (1991).

[3] A. L. Williams, et al, Phys. Rev. C 44, 2025 (1991).

[4] E. Oset and D. Strottman, preprint.

[5] R. Gilman, et al, Phys. Rev. C 29, 2395 (1984).

[6] R. Gilman, et al, Phys. Rev. C 32, 349 (1985); Phys. Rev. C 34, 1895 (1986).

[7] H. T. Fortune and R. Gilman, Phys. Rev. C 33, 2171 (1986).

[8] M. Johnson, Proceedings of the Second International Conference on Medium and High Energy Nuclear Physics, Taiwan, 1990, p. 329 (North-Holland Publishing Co., 1991).

[9] N. Auerbach, et al, Phys. Rev. Lett. 59, 1076 (1987); Phys. Rev. C 38, 1277 (1988).

[10] M. Leitch, in Proceedings of the Second LAMPF International Workshop on Pion-Nucleus Double Charge Exchange, 1989 (World Scientific, 1990), p. 27.

[11] E. Oset and D. Strottman, private communication.

[12] Q. Haider and L. C. Liu, Phys. Rev. C 36, 1636 (1987).

[13] M. K. Jones et al., Phys. Rev. C46, 52 (1992); C42, R807 (1990).

[14] G. S. Kyle, et al, Phys. Rev. Lett. 52, 974 (1984).

[15] T. Takaki and M. Thies, Phys. Rev. C 38, 2230 (1988).

[16] R. Schiavilla, V. R. Pandharipande, and R. B. Wiringa, Nucl. Phys. A449, $21.9(1986)$.

[17] S. Ritt, et al, Phys. Rev. C 43, 745 (1991).

[18] W. B. Kaufmann, P. B. Siegel, and W. R. Gibbs (to be published).

[19] Y.-F. Yen, et al, Phys. Rev. Lett. 66, 1959 (1991).

[20] A. Erell et al., Phys. Rev. C34, 1822 (1986). 
[21] W. M. Alberico, M. Ericson, and A. Molinari Nucl. Phys. A379, 429 (1982).

[22] T. Udagawa, S. W. Hong, and F. Osterfeld, Phys. Lett. 245B,1 (1990).

[23] R. S. Hicks, J. Button-Shafer, B. Debebe, J. Dubach, A. Hotta, R. L. Huffman, R. A. Lindgren, and G. A. Peterson, Phys. Rev. Lett. 60, 905 (1988).

[24] G.E. Brown, et al, Phys. Lett. B118, 39 (1982).

[25] B. Schwesinger, et al, Phys. Lett. B132, 269 (1983).

[26] M. Steinacher, et al, Nucl. Phys. A517, 413 (1991).

[27] R. Ransome, et al, Phys. Rev. C 42, 1500 (1990)

[28] S. Hyman, et al, Phys. Rev. C 41, R409 (1990).

[29] W.J. Burger et al, Phys. Rev. Lett. 57, 58 (1986).

[30] C.H.Q. Ingram et al, Phys. Rev. C 27, 1578 (1983).

[31] E.R. Kinney, et al, Phys. Rev. Lett. 57, 3152 (1986); P. Gram, Nucl. Phys. A527, 45c-85c (1991).

[32] K. Masutani and Y. Yazaki, Nucl. Phys. A407, 309 (1983).

[33] R. Tacik et al, Phys. Rev. C 40, 256 (1989).

[34] W. Bruckner et al, Nucl. Phys. A469, 617 (1987).

[35] P. B. Siegel and W. R. Gibbs, Phys. Rev. C 36, 2473 (1987).

[36] N. S. Chant and P. G. Roos, Phys. Rev. C 38, 787 (1989).

[37] K. K. Seth, et al, Phys. Rev. C 41, 2800 (1990).

[38] J. A. Carr, H. McManus, and K. Stricker-Bauer, Phys. Rev. C 36, 1472 (1987).

[39] D. H. Wright, et al, Phys. Rev. C 36, 2139 (1987); Phys. Rev. C 37, 1155 (1988).

[40] A. Altmann, et al, Phys. Rev. Lett. 50, 1187 (1983).

[41] T.-S. H. Lee, K. Ohta, and M. Thies, Ann. Phys. 163, 420 (1985). 
[42] B.G. Ritchie, N.S. Chant, and P.G. Roos, Phys. Rev. C 30, 969 (1984); Phys. Rev. C 32, 334 (1985).

[43] D. Mack, Ph.D. dissertation, University of Maryland, 1987 (unpublished).

[44] S. Hyman, Ph.D. dissertation, University of Maryland, 1989 (unpublished).

[45] D. Mack, et al, Phys. Rev. C 45, 1767 (1992).

[46] D. Zhang, Ph.D. dissertation, University of Maryland, 1990 (unpublished).

[47] M. Baumgartner, et al, Phys. Lett. B112, 35 (1982); Nucl. Phys. A398, 451 (1983).

[48] M. Khandaker, Ph.D. dissertation, University of Washington, 1988 (unpublished).

[49] R. Hamers, Ph.D. thesis, Free University of Amsterdam, The Netherlands, 1989 (unpublished).

[50] Th.S. Bauer et al submitted to Phys, Rev. C.

[51] F. Adimi, et al, Phys. Rev. C 45, 2589 (1992).

[52] For a discussion of the current status of the physics related to the sigma term, see the series of $\pi N$ Newsletters, edited by R. E. Cutkosky, et al, who can supply copies. See also J. Gasser, H. Leutwyler, and M. E. Sainio, Phys. Lett. B 353, 252 (1991).

[53] O. Häusser, Seventh International Conference on Polarization in Nuclear Physics, Paris, 1990 (Colloque de Physique 51, C6-99 (1990)).

[54] O. Häusser, et al, Invited Talk, Proceedings of the Fourth Conferevee on the Intersections between Particle and Nuclear Physics, 1991, Tucson, Arizona.

[55] B. Larson, et al, Phys. Rev. Lett. 67, 3356 (1991).

[56] B. Larson, et al, Phys. Rev. A 44, 3108 (1991).

[57] R. Tacik, et al, Phys. Rev. Lett. 63, 1784 (1989).

[58] R. Mach and S. S. Kamalov, Nucl. Phys. A511, 601 (1990).

[59] S. Chakravarti, private communication. 
[60] R. A. Brandenburg, Y. E. Kim, and A. Tubis, Phys. Rev. C 12, 1368 (1975).

[61] J. L. Friar, et al, Phys. Rev. C 34, 1463 (1986); W. R. Gibbs, private communication.

[62] D. R. Tilley, H. R. Weller, and H. H, Hasan, Nucl. Phys. A474, 22 (1987).

[63] C. Bennhold, B. K. Jennings, L. Tiator, and S. S. Kamalov (to be published).

[64] A. Doron, et al, Phys. Rev. Lett. 48, 989 (1982); Phys. Rev. C 26, 1604 (1982).

[65] J. Görgen, et al, Phys. Rev. Lett. 66, 2193 (1991).

[66] D. Dehnhard et al, Phys. Rev. Lett. 43, 1578 (1979); S. J. Seestrom-Morris, et al, Phys. Rev. C 28, 1301 (1983).

[67] W. R. Gibbs, private communication.

[68] W. R. Gibbs and J.-P. Dedonder, Phys. Rev. C 46, 1825 (1992).

[69] M. Hirata, F. Lenz, and M. Thies, Phys. Rev. C 28, 785 (1983).

[70] J. A. Faucett, et al, Phys. Rev. C 30, 1622 (1984).

[71] S. Gilad, et al, Phys. Rev. Lett. 57, 2637 (1986).

[72] F. Lenz, Nucl. Phys. A478, 581c (1988).

[73] Ferreira and Dosch, Phys. Rev. C 40, 1750 (1989).

[74] J. Kuti and W. W. Hughes, Ann. Rev. of Nucl. and Part. Sci. 33, 611 (1983).

[75] M. H. A'nmed and G. G. Ross, Nucl. Phys. B111, 441 (1976).

[76] J. Kodaira, et al, Nucl. Phys. B159, 99 (1979).

[77] G. R. Farrar and D. R. Jackson, Phys. Rev. Lett. 35, 1416 (1975).

[78] J. D. Bjorken, Phys. Rev. 148, 1457 (1966); Phys. Rev. D 1, 136 ? (1970).

[79] J. Ellis and R. L. Jaffe, Phys. Rev. D 9, 1444 (1974).

[80] M. J. Alguard, et al, Phys. Rev. Lett. 37, 1261 (1978); 41, 70 (1978). G. Baum, et al, Phys. Rev. Lett. 51, 1135 (1981).

[81] J. Ashman, et al, Phys. Lett. B206, 364 (1988). 


\section{Publications}

The following is a list of publications of the NMSU group between October 1, 1991, and December 31, 1992, not previously reported. The names of authors supported by DOE under this grant while the work reported was done are in bold type.

\subsection{Published Papers}

1. Mass Dependence of High-Energy Pion Double Charge Exchange, A. L. Williams, K. W. Johnson, G. P. Kharimanis, H. Ward, C. F. Moore, J. A. McGill, C. L. Morris, G. R. Burleson, J. A. Faucett, M. Rawool-Sullivan, D. S. Oakley, M. Burlein, H. T. Fortune, E. Insko, R. Ivie, J. M. O'Donnell, and D. Smith; Phys. Rev. C 44, 2025 (1991).

2. Elastic Scattering of $\pi^{+}$and $\pi^{-}$from ${ }^{4} \mathrm{He}$ Between 90 and $240 \mathrm{MeV}, \mathrm{B}$. Brinkmöller, C. L. Blilie, D. Dehnhard, M. Jones, G. M. Martinez, S. K. Nanda, S. M. Sterbenz, Yi-Fen Yen, L. G. Atencio, S. J. Greene, C. L. Morris, S. J. Seestrom, G. R. Burleson, K. S. Dhuga, J. A. Faucett, R. W. Garnett, K. Maeda, C. F. Moore, S. Mordechai, A. Williams, S. H. Yoo, and L. C. Bland; Phys. Rev. C 44, 2031 (1991).

3. A Neutron Hodoscope for Medium Energy np Scattering Experiments, R. Garnett, D. Grosnick, K. Johnson, D. Lopiano, A. Rask, T. Shima, H. Spinka, R. Stanek, D. Underwood, A. Yokosawa, M. Beddo, G. Burleson, J. A. Faucett, G. Kyle, M. Devereux, G. Glass, S. Nath, J. Jarmer, S. Penttilä, R. Jeppesen, and G. Tripard; Nucl. Instr. and Methods A309, 508 (1991).

4. A New Multiplexing Scheme for Cathode-Strip Readout Chambers, M. W. RawoolSullivan, J. F. Amann, L. G. Atencio, R. L. Boudrie R. Lomax, C. L. Morris, M. Murray, and R. M. Whitton; Mucl. Instr. and Methods A311, 168 (1992).

5. A Simple Three-Dimensional Magnetic Field Interpolation Technique, R. W. Garnett and G. R. Burleson; Nucl. Instr. and Methods A313, 501 (1992).

6. Accelerator Measurements of NaI Response to Medium Energy Neutrons and Application to a Satellite-Borne Spectrometer, P. P. Dunphy, E. L. Chupp, M. Popecki, D. J. Forrest, D. Lopiano, T. Shima, H. Spinka, G. Glass, G. Burleson, and M. Beddo; Exp. Astronomy 2, 233 (1992).

7. Neutron-Proton Elastic Scattering Spin-Spin Parameter Measurements between 500 and $800 \mathrm{MeV}$ : I. $C_{S L}$ and $C_{L L}$ Measurements at Backward c.m. Angles, W. R. Ditzler, D. Hill, J. Hoftiezer, K. F. Johnson, D. Lopiano, T. Shima, H. Shimizu, H. Spinka, R. Stanek, D. Underwood, R. G. Wagner, A. Yokosawa, G. R. Burleson, 
J. A. Faucett, C. A. Fontanla, R. W. Garnett, C. Luchini, M. W. RawoolSullivan, T. S. Bhatia, G. Glass, J. C. Hiebert, R. Kenefick, S. Nath, L. C. Northcliffe, R. Damjanovich, J. J. Jarmer, J. Vaninetti, R. H. Jeppesen, and G. E. Tripard; Phys. Rev. D 46, 2792 (1992).

8. Dominance of the Two-Nucleon Mechanism in ${ }^{16} \mathrm{O}\left(\pi^{+}, p p\right)$ at $115 \mathrm{MeV}, \mathrm{D}$. J. Mack, P. G. Roos, H. Breuer, N. S. Chant, S. D. Hyman, F. Khazie, B. G. Ritchie, J. D. Silk, G. S. Kyle, P. A. Amaudruz, Th. S. bauer, C. H. Q. Ingram, D. Renker,

R. A. Schumai:her, U. Sennhauser, and W. J. Burger, Phys. Rev. C 45, 1767 (1992).

9. Two-Nucleon Absorption of $\pi^{+}$in ${ }^{4} \mathrm{He}$ at $T_{\pi^{+}}=114$ and $162 \mathrm{MeV}, \mathrm{F}$. Adimi, $\mathrm{H}$. Breuer, B. S. Flanders, M. A. Khandaker, M. G. Khayat, P. G. Roos, D. Zhang, Th. Bauer, J. Konijn, C. T. A. M. de Laat, G. S. Kyle, S. Mukhopadhyay, M. Wang, and R. Tacik; Phys. Rev. C 45, 2589 (1992).

\subsection{Papers Submitted for Publication}

1. Neutron-Proton Elastic Spin-Spin Correlation Parameter Measurements between 500 and $800 \mathrm{MeV}$ : II. $C_{S S}$ and $C_{L S}$ at Forward c.m. Angles, T. Shima, D. Hill, K. F. Johnson, H. Shimizu, H. Spinka, R. Stanek, D. Underwood, A. Yokosawa, G. Glass, J. C. Hiebert, R. A. Kenefic, S. Nath, L. C. Northcliffe, G. R. Burleson, R. W. Garnett, J. A. Faucett, M. W. Rawool-Sullivan, R. Damjanovich, J. J. Jarmer, R. H. Jeppesen, and G. E. Tripard; submitted to Phys. Rev. D.

2. Search for an $\eta$ Bound State in Pion DCX on ${ }^{18} O$, J. D. Johnson, G. R. Burleson, C. Edwards, M. El-Ghossain, M. A. Espy, R. Garnett, A. Hussein, K. Johnson, C. F. Moore, C. L. Morris, J. M. O'Donnell, M. Palarczyk, M. Rawool-Sullivan, D. Watson, C. Whitley, and A. L. Williams; submitted to Phys. Rev. C.

3. The ${ }^{10} B(p, n){ }^{10} C$ Rection at $186 \mathrm{MeV}$, L. Wang, J. Rapaport, X. Yang, S. DeLucia, B. Luther, D. Marchlenski, E. Sugarbaker, C. Foster, C. D. Goodman, Y. Wang, L. Rybarcyk, T. N. Taddeucci, D. Lindgren, B. K. Park; submitted to Phys. Rev. C.

\subsection{Papers Presented at Meetings and Conferences}

At the Spring Meeting of the American Physical Society, Washington, DC, April 20-24, 1992:

1. $A_{n n}$ for $500 \mathrm{MeV} \vec{p}+\vec{p}$ Elastic Scattering, G. Hoffmann, M. Barlett, W. Kielhorn, G. Pauletta, M. Purcell, L. Ray, J. Amann, J. Jarmer, K. Jones, S. Penttilä, N. Tanaka, G. Burleson, J. Faucett, M. Gilani, L. Stevens, A. Mack, D. Milaildis, T. Averett, J. Comfort, J. Görgen, and J. Tinsley; Bull. Am. Phys. Soc. 37, 903 (1992). 
2. Pion Absorption in ${ }^{4} \mathrm{He}, \mathrm{F}$. Adami, M. G. Khayat, H. Breuer, B. S. Flanders, M. Khandaker, P. G. Roos, D. Zhang, Th. S. Bauer, J. Konijn, C. T. A. M. de Laat, G. Kyle, S. Hukhopadhyay, M. Wang, and R. Tacik; Bull. Am. Phys. Soc. 37, 915 (1992).

3. Pion Double Charge Exchange on ${ }^{16} O$ Above the Resonance, D. P. Beatty, R. Gilman, Q. Sun, H. T. Fortune, A. L. Williams, D. Smith, P. Hui, S. Loe, M. A. Landau, M. Rawool-Sullivan, J. Urbina, J. M. O'Donnell, and D. L. Watson; Bull. Am. Phys. Soc. 37, 916 (1992).

4. The Search for the Eta Component of Pion Double Charge Exchange, J. D. Johnson, C. F. Moore, K. W. Johnson, H. Ward, C. Whitley, A. Hussein, R. W. Garnett, L. C. Liu, C. L. Morris, J. M. O'Donnell, G. R. Burleson, M. W. RawoolSullivan, M. El-Ghossain, D. Dehnhard, C. Edwards, M. A. Espy, M. Palarczyk, A. L. Williams, and D. L. Watson; Bull. Am. Phys. Soc. 37, 916 (1992).

5. Pion-Nucleus Elastic Scattering at Energies above the $\Delta$ (1292) Resonance, $M$. W. Rawool-Sullivan, J. A. Faucett, G. R. Burleson, K. W. Johnson, G. P. Kharimanis, A. L. Williams, C. Whitley, C. F. Moore, J. A. McGill, C. L. Morris, D. J. Ernst, K. S. Dhuga, E. Insko, J. M. O'Donnell, and H. T. Fortune; Bull. Am. Phys. Soc. 37, 916 (1992).

6. High-Energy Pion Double Charge Exchange on ${ }^{16}$ O, D. P. Beatty, M. RawoolSullivan, G. R. Burleson, M. El-Ghossain, H. T. Fortune, A. L. Williams, D. Smith, D. L. Morris, R. Garnett, J. Johnson, H. Ward, C. Whitley, S. K. Matthews, M. Palarczyk, C. Edwards, M. Espy, and D. Watson; Bull. Am. Phys. Soc. 37, 916 (1992).

At the Second US-Japan Symposium on Pion Physics Above the Delta, Loa Alamos, NM, August, 1992:

1. Measurements of DCX at LAMPF, 300-500 MeV, G. R. Burleson, invited paper.

At the Fall Meeting of the Division of Nuclear Physics of the Americal Physical Society, October 14-17, 1992:

1. The Search for the Eta Component of Pion Double Charge Exchange, J. D. Johnson, C. F. Moore, K. W. Johnson, H. Ward, C. Whitley, A. Hussein, R. W. Garnett, L. C. Liu, C. L. Morris, J. M. O'Donnell, G. R. Burleson, M. W. RawoolSullivan, M. El-Ghossain, D. Dehnhard, C. Edwards, M. A. Espy, M. Palarazyk, A. L. Williams, and D. L. Watson; Bull. Am. Phys. Soc. 37, 1292 (1992). 
2. ${ }^{4} \mathrm{He}\left(\pi^{+}, p p d\right)$ at $114 \mathrm{MeV}, \mathrm{F}$. Adimi, M. G. Khayat, H. Breuer, B. S. Flanders, M. Khandaker, P. G. Roos, D. Zhang, Th. S. Bauer, J. Konijn, C. T. A. M. de Laat, G. Kyle, S. Mukhopadhyay, M. Wang, and R. Tacik; Bull. Am. Phys. Soc. 37, 1303 (1992).

3. Large Solid Angle Measurements of the Pion-Nucleus Absorption Cross Section in the Region of the $\Delta$ Resonance, N. Simicevic, W. Fong, N. K. Gregory, R. P. Redwine, D. Rowntree, K. E. Wilsin, G. Backenstoss, J. Köhler, M. Krödel, A. Lehmann, H. J. Weyer, M. Wildi, R. Schumacher, T. Alterholz, A. Hoffart, H. Ullrich, P. A. M. Gram, H. Breuer, F. D. Smit, G. Kyle, Z. Lin, S. Mukhopadhyay, M. H. Wang, G. van der Steenhoven, T. Dooling, A. Klein, H. Döbbeling, Q. Ingram, K. Koch, B. Kotlinski, G. Mahl, M. Michaelian, U. Sennhauser, D. Bosnar, R. Brkovic, M. Furic, and T. Petkovic; Bull. Am. Phys. Soc. 37, 1303 (1992). 


\section{Personnel}

The effort that has been devoted to this grant by those supported by it, from April 1, 1992, through March 31, 1993 (projected) is given below.

Faculty Members:

George R. Burleson, 6 man-months

Gary S. Kyle, 6 man-months

Postdoctoral Research Associates:

Maozhi Wang, 12 man-months

Brent K. Park, 12 man-months

\section{Graduate Students:}

Zhinan Lin, 12 man-months

Qihua Zhao, 12 man-months

Tinghua Chang, 10 man-months

Sean P. Blanchard, 3 man-months

Brian A. Lail, 3 man-months

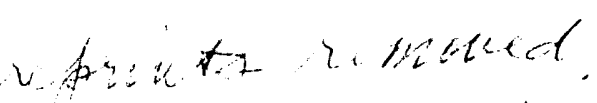

$$
\begin{aligned}
& \cot x
\end{aligned}
$$



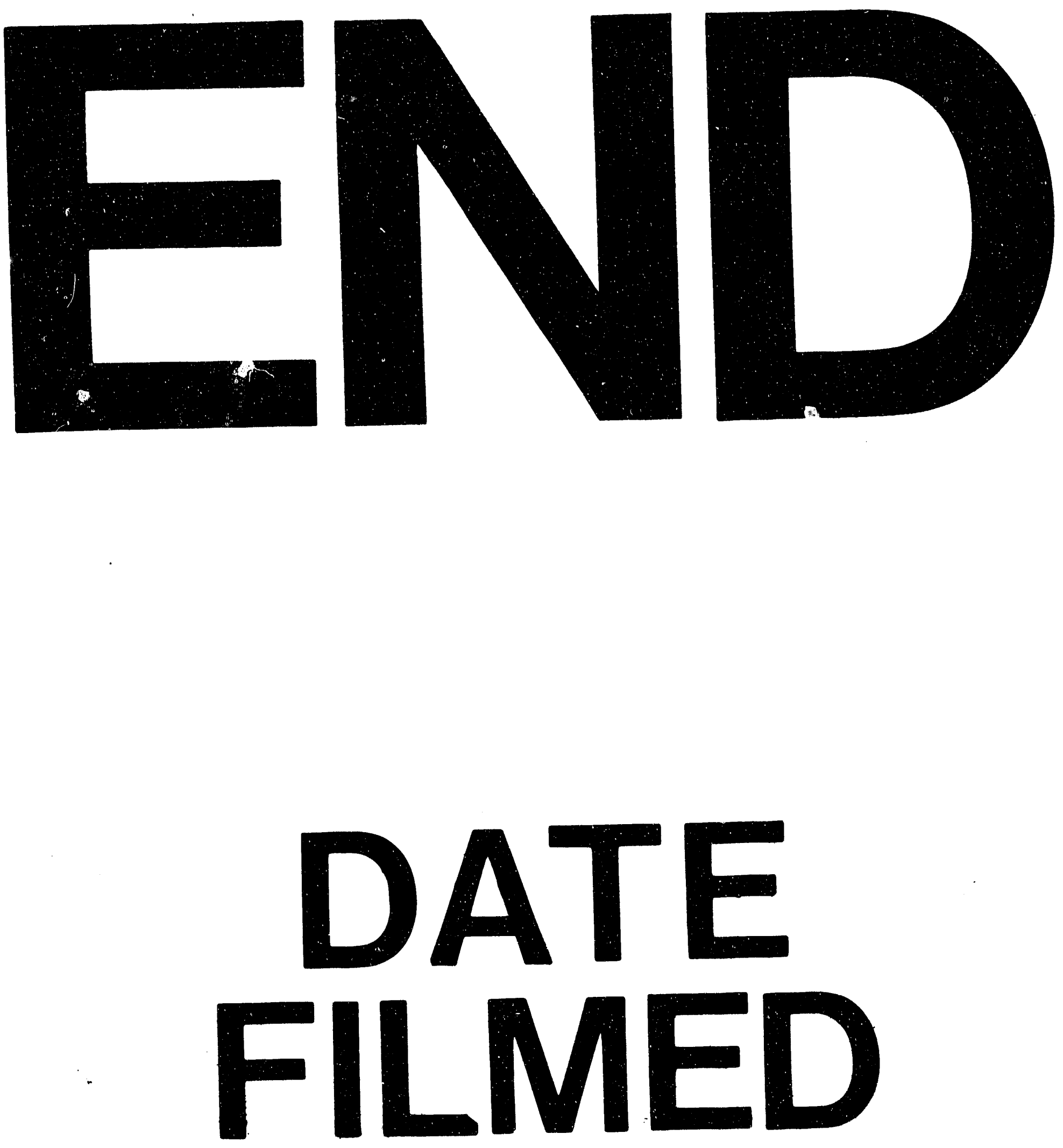

I

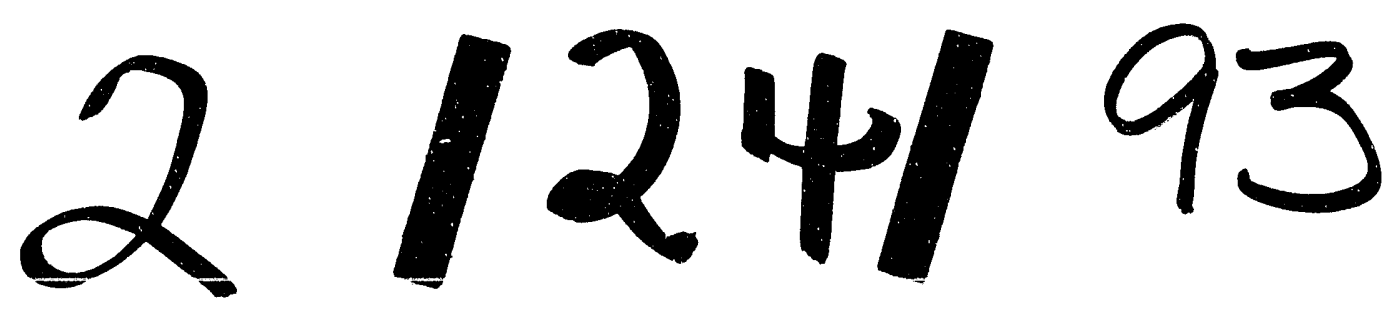


